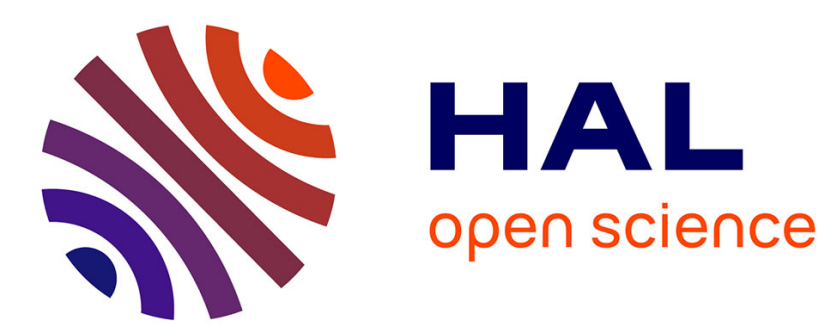

\title{
Analyticity of solutions to thermo-elastic-plastic flow problem with microtemperatures
}

Moncef Aouadi, Mohamed Ben Bettaieb, Farid Abed-Meraim

\section{To cite this version:}

Moncef Aouadi, Mohamed Ben Bettaieb, Farid Abed-Meraim. Analyticity of solutions to thermoelastic-plastic flow problem with microtemperatures. Journal of Applied Mathematics and Mechanics / Zeitschrift für Angewandte Mathematik und Mechanik, 2021, 33 p. 10.1002/zamm.202000346. hal-03254877

\section{HAL Id: hal-03254877 \\ https://hal.science/hal-03254877}

Submitted on 9 Jun 2021

HAL is a multi-disciplinary open access archive for the deposit and dissemination of scientific research documents, whether they are published or not. The documents may come from teaching and research institutions in France or abroad, or from public or private research centers.
L'archive ouverte pluridisciplinaire HAL, est destinée au dépôt et à la diffusion de documents scientifiques de niveau recherche, publiés ou non, émanant des établissements d'enseignement et de recherche français ou étrangers, des laboratoires publics ou privés. 


\title{
Analyticity of solutions to thermo-elastic-plastic flow problem with microtemperatures *
}

\author{
Moncef Aouadi $^{1, *}$, Mohamed Ben Bettaieb ${ }^{2, * *}$, Farid Abed-Meraim ${ }^{2, * * *}$ \\ ${ }^{1}$ Université de Carthage, Ecole Nationale d'Ingénieurs de Bizerte, 7035, BP66, Tunisia \\ ${ }^{2}$ Arts et Metiers Institute of Technology, CNRS, \\ Université de Lorraine, LEM3, F-57000, Metz, France
}

\begin{abstract}
In this paper, we study some qualitative and numerical properties of new equations including the coupled effects of thermal elastic-plastic theory with microtemperatures. We establish the necessary and sufficient conditions to guarantee that the model dissipates energy. The one-dimensional case, which corresponds to isotropic hardening problem, is chosen in order to present some qualitative and numerical properties. With the help of the semigroup theory of linear operators, we prove the well-posedness of the one-dimensional problem corresponding to plastic flow. Then, we show that the associated $C_{0}$-semigroup is not analytical in general, except for a special case. The exponential stability of the solutions is kept in all cases. Finally, a numerical tool, based on the finite element method, is developed to validate the proposed model and to show its capability. Particular attention is paid to the consideration of the elastoplastic behavior in the development of this tool.
\end{abstract}

Keywords: elasto-plastic, gradient-dependent, microtemperatures, well-posedness, exponential stability, finite element analysis.

\section{$1 \quad$ Introduction}

The classical thermo-gradient-dependent plasticity theory combines the strain gradient elastoplastic behavior of homogeneous body with heat conduction governed by Fourier's law (see e.g. [1, 6]). To be more realistic, for such microstructured materials, the influence of microstructure should be taken into account both for the elastoplastic deformation as well as for the temperature distribution. For this, we derive in this paper new governing equations

\footnotetext{
${ }^{*}$ Corresponding author E-mail: Moncefaouadi00@gmail.com

** E-mail: Mohamed.BenBettaieb@ensam.eu

*** E-mail: Farid.Abed-Meraim@ensam.eu
} 
for thermo-gradient-dependent theory of plasticity including the coupled effects of thermal elastoplastic theory with microtemperatures. The interest of microtemperatures and gradient theories is stimulated by the fact that both are adequate to investigate important problems related to size effects and nanotechnology. Moreover, the experimental observations have shown that the classical continuum theories cannot be used to satisfactorily describe some phenomena. Both facts represent an important improvement in the perspective of real applications in many engineering and geophysics applications and also in nanomanufactured activities. This gives rise to a large variety of generalized continuum models based on well-established continua [3, 39, as well as on more recent strain-gradient plasticity approaches to avoid the difficulties in localization simulation of single phase materials. In this context, gradient-dependent models have been recognized by several authors to provide a satisfactory framework for the analytical and numerical analysis of strain localization in single phase solids.

In this paper, we include both the second-order strain gradient terms in the stress-strain law and the microtemperatures concept into the second law of thermodynamics. Microtemperatures depend homogeneously on microcoordinates of the microelements, which are based on the microstructure of the continuum. The concept of microtemperatures has been introduced for the first time by Wozniak in [37, 38]. He considered in [38. that the continuum (macromedium) is composed of particles $X$ with macrocoordinates. Each particle is assumed at the same time as the origin of the so-called system of spatial local microcoordinates $\left(x_{1}, x_{2}, x_{3}\right)$. The spatial coordinates $x_{i}^{\prime}$ of the point $X^{\prime}$ of the microelement $\omega_{m}$ are represented in the form $x_{i}^{\prime}=x_{i} \psi_{i k} \xi_{k}$, where $x_{i}$ are the spatial coordinates of the centroid $X$ of the microelement $\omega ; X_{k}^{\prime}$ and $X_{k}$ are the material coordinates of $X^{\prime}$ and $X$, and $\xi_{k}=X_{k}^{\prime}-X_{k}$. The functions $\psi_{i k}$ are called microdeformations. Grot [16] extended the thermodynamics of a continuum with microstructure to microelements to describe the concept of microtemperatures. According to Grot [16], the microtemperatures should be determined from a balance law. By adding the first order moment of the energy equations to the usual balance laws, the Clausius-Duhem inequality is modified to include microtemperatures.

We denote by $X$ the center of mass of a generic microelement $\omega_{m}$ and $X^{\prime}$ an arbitrary point in the microelement. In [16], the inverse of the temperature $\Theta^{\prime}$ at the point $X^{\prime}$ of the microelement $\omega_{m}$ is a linear function of the local microcoordinates $\xi_{k}$

$$
\frac{1}{\Theta^{\prime}}=\frac{1}{\Theta(X)}+\frac{1}{\Theta_{k}(X)} \xi_{k}
$$

where $\Theta$ is the temperature at the centroid $X$ and $\frac{1}{\Theta_{k}}, k=1,2,3$, form a vector that represents temperature variation within the microelement $\omega_{m}$ according to equality 1.1. After multiplying by the product $\Theta \Theta^{\prime}$, equality (1.1) can be rewritten as

$$
\Theta^{\prime}=\Theta+\tau_{k} \xi_{k} \quad \text { with } \quad \tau_{k}=-\frac{1}{\Theta_{k}} \Theta \Theta^{\prime}, \quad k=1,2,3
$$

Grot [16] takes $\Theta\left(x_{1}, x_{2}, x_{3}\right)$ and $\bar{\Theta}_{k}\left(x_{1}, x_{2}, x_{3}\right):=\frac{1}{\Theta_{k}} \Theta$ as unknown functions, while Ieşan and Quintanilla [18, $19]$ take $\Theta\left(x_{1}, x_{2}, x_{3}\right)$ and $T_{k}=-\frac{\tau_{k}\left(x_{1}, x_{2}, x_{3}\right)}{\Theta\left(x_{1}, x_{2}, x_{3}\right)}, k=1,2,3$ as unknown functions. The vectors $\left(\bar{\Theta}_{1}, \bar{\Theta}_{2}, \bar{\Theta}_{3}\right)$ and $\left(T_{1}, T_{2}, T_{3}\right)$ are called the microtemperature vectors by Riha [31] and Ieşan and Quintanilla [18, 19, respectively. 
Clearly, neither so called microtemperature vector nor its components are temperatures, they represent the variation of the temperature within a microelement $\omega[22$.

The effect of microtemperature has been studied first on thermoelastic continua [8, 7, 11, 18, 19, 20, 21, 26. Experimental data for the silicone rubber containing spherical aluminium particles and for human blood were found to conform closely to the predicted theoretical thermal conductivity.

Our first main objective is to derive the thermomechanical theory for gradient-dependent plastic materials with microtemperatures effects. One can find many propositions in the literature for the introduction of thermodynamics into plasticity. More recently, the thermal effects have been introduced in relevant strain gradient plasticity models within the thermodynamically consistent framework (see e.g. [1, 6, 33, 34, 35, 36]); but the microtemperatures effects were not addressed. In most developed models, the higher-order thermo-mechanical gradient plasticity theory is developed within the thermodynamically consistent framework based on the concept of thermal activation energy and dislocations interaction mechanisms, and the decomposition of the thermodynamic microforces into energetic and dissipative counterparts. The present work, which has not been obtained in any reference yet, is the first work where the elastoplastic deformation is considered together with thermal and microtemperatures deformation in the frame of strain gradient theory. The development of such a model within rigorous thermodynamics is essential, to put in evidence a variety of phenomena that needed explanation with valuable applications in several fields of technology.

Our second main objective is to show that the $C_{0}$-semigroup associated with the derived equations in one dimensional setting is analytic or exponentially stable. Our intention is to show how the dissipation mechanism, due to temperature and microtemperatures effects, implies several qualitative properties. For this, we are interested in knowing the conditions that the resulting constitutive coefficients have to satisfy in order to get the internal energy and the dissipation to be positive definite functions. Thanks to these dissipations, we will prove that generically the coupling is so strong that the thermal together with the microtemperatures dissipations make the solutions analytic. This is an important fact. Two consequences of this fact are the exponential decay and the impossibility of localization in time of the solutions. To our best knowledge, the analyticity of solutions issue has never been studied for thermo-elastoplastic problems, whereas it has been widely treated for thermoelastic problems (see e.g. 4, 5, 27]).

This paper is organized as follows. In Section 2, we derive the governing equations for thermo-gradientdependent theory of plasticity including the microtemperatures effects. To demonstrate the salient feature of the gradient-dependent model of plasticity with microtemperatures, the one-dimensional counterpart of the derived model is detailed in Section 3. Thus, linear theory is applicable and the necessary and sufficient conditions guaranteeing the dissipation of energy are established. In Section 4 , using the $C_{0}$-semigroup theory, we prove the well-posedness of the problem corresponding to plastic flow. In Section 5, we prove that the associated semigroup is not analytical in general, except for a special case. The exponential stability of the solutions is kept in all cases. Section 6 is devoted to the presentation of an implicit finite element tool, specifically developed to integrate the derived constitutive equations and to achieve the corresponding numerical simulations. 


\section{Notations and conventions}

The main notations and conventions used in this paper are clarified in the box bellow. Additional notations will be provided when needed.

$f_{x} \quad$ partial derivative of $f$ with respect to $x$.

$f_{x x} \quad$ second-order partial derivative of $f$ with respect to $x$.

$\dot{f} \quad$ derivative of $f$ with respect to time.

$f \cdot g \quad$ inner product.

$f: g \quad$ double contraction product.

$\delta f \quad$ virtual field of $f$.

$\nabla f \quad$ gradient of $f$.

$\operatorname{div} f$ divergence of $f$.

$\Delta f \quad$ Laplacian of $f$.

\section{Thermodynamic framework}

Few attempts to derive the thermodynamic formulation of the generalized continuum mechanics exist in the literature, especially including the microtemperatures effects. In this paper, we derive the well-suited thermomechanical coupling with microtemperatures. Therefore, the principle of virtual power states that given any sub-body $V$, the total virtual power of the external, internal and inertia forces is zero in any admissible virtual state of motion. This principle can be mathematically expressed as follows:

$$
\int_{V}(\operatorname{div} \boldsymbol{\sigma}+\mathbf{b}-\rho \ddot{\mathbf{u}}) \cdot \delta \dot{\mathbf{u}} d v+\int_{\partial V}(\mathbf{t}-\boldsymbol{\sigma} \cdot \mathbf{n}) \cdot \delta \dot{\mathbf{u}} d a=0
$$

where $\boldsymbol{\sigma}$ is the Cauchy stress tensor, $\mathbf{b}$ is the volume force vector, $\mathbf{u}$ is the displacement vector, $\mathbf{t}$ is the surface traction vector, $\rho$ is the mass density and $\mathbf{n}$ is the outward unit normal to $\partial V$. The virtual velocity field $\delta \dot{\mathbf{u}}$ may be arbitrarily specified if and only if

$$
\begin{aligned}
& \operatorname{div} \boldsymbol{\sigma}+\mathbf{b}=\rho \ddot{\mathbf{u}}, \\
& \mathbf{t}=\boldsymbol{\sigma} \cdot \mathbf{n} .
\end{aligned}
$$

Eq. 2.3$)_{1}$ expresses the local static $(\rho \ddot{\mathbf{u}}=\mathbf{0})$ or dynamic $(\rho \ddot{\mathbf{u}} \neq \mathbf{0})$ equilibrium or balance force; while Eq. 2.3$)_{2}$ defines the traction boundary condition.

In this paper, the second law of thermodynamics is modified in order to introduce the concept of microtemperatures and the first order moment of the energy is added to the usual balance law. Here we have two principles of thermodynamics:

(i) the conservation of energy, namely

$$
\rho \dot{e}=\boldsymbol{\sigma}: \dot{\varepsilon}+\operatorname{div} \mathbf{q}+\rho v
$$

where $e$ is the specific internal energy, $\mathbf{q}$ is the heat flux vector, $v$ is the heat supply and

$$
\varepsilon=\frac{1}{2}\left(\operatorname{grad} \mathbf{u}+(\operatorname{grad} \mathbf{u})^{T}\right),
$$


is the strain tensor;

(ii) the balance of first moment of energy [16, 37, 38,

$$
\rho \dot{\boldsymbol{\Lambda}}=\operatorname{div}(\mathbf{Q})+\mathbf{q}-\boldsymbol{\varsigma}+\rho \boldsymbol{\mu},
$$

where $\boldsymbol{\Lambda}$ is the first moment of energy vector, $\mathbf{Q}$ is the first heat flux moment second-rank tensor, $\varsigma$ is the microheat flux average and $\boldsymbol{\mu}$ is the first moment of the heat source vector.

Following Grot [16] and Eringen [12, we wish to extend the thermo-gradient-dependent theory of plasticity derived by Aouadi et al. [6] by taking into account the microtemperatures effect of the particles. This can be done by deriving the balance laws 2.3 -2.6 to the case in which the temperatures of the particles are different. In the spirit of their works, let us assume that $\mathbf{X}$ be the center of mass of a generic microelement in the reference configuration. We assume that deriving the particle temperature $T^{\prime}$ has the form [16]

$$
T^{\prime}\left(\mathbf{X}^{\prime}, t\right)=T(\mathbf{X}, t)+\mathbf{T}(\mathbf{X}, t) \cdot \boldsymbol{\xi}
$$

where $\boldsymbol{\xi}=\mathbf{X}^{\prime}-\mathbf{X}, T$ is the absolute temperature and the function $\mathbf{T}$ is called microtemperatures. The variables in 2.7) form a vector and represent the variation of the temperature within a microvolume. They will be considered as independent thermodynamic variables to be determined by the balance laws.

For each microelement, the microentropy $S$ satisfies the inequality [29]

$$
\rho^{\prime} \dot{S}^{\prime}-\operatorname{div}\left(\frac{\mathbf{q}^{\prime}}{T^{\prime}}\right)-\frac{\rho^{\prime} v^{\prime}}{T^{\prime}} \geq 0
$$

The macroscopic second law is derived for 2.8 by integrating over a volume $d v$. From (2.7) the average of (2.8) yields the inequality [16]

$$
\frac{d}{d t} \int_{V} \rho S d v-\int_{\partial V}\left(\frac{\mathbf{q}}{T}+\frac{\mathbf{Q}}{T} \cdot \mathbf{T}\right) d \mathbf{a}-\int_{V} \rho\left(\frac{v}{T}+\frac{\mathbf{T}}{T} \cdot \boldsymbol{\mu}\right) d v \geq 0 .
$$

The inequality (2.9), in the usual way, leads to the differential inequality [16]

$$
\rho \dot{S}-\operatorname{div}\left(\frac{\mathbf{q}}{T}+\frac{\mathbf{Q}}{T} \cdot \mathbf{T}\right)-\rho\left(\frac{v}{T}+\frac{\mathbf{T}}{T} \cdot \boldsymbol{\mu}\right) \geq 0 .
$$

By eliminating $v$ and $\boldsymbol{\mu}$ from 2.10 through the use of 2.4 and (2.6), we obtain the inequality

$$
\rho(T \dot{S}-\dot{e}-\mathbf{T} \cdot \dot{\boldsymbol{\Lambda}})+\boldsymbol{\sigma}: \dot{\varepsilon}+\frac{\mathbf{q}}{T} \cdot \nabla T+\frac{\nabla T}{T} \cdot \mathbf{Q} \cdot \mathbf{T}-\mathbf{Q}: \nabla \mathbf{T}+(\mathbf{q}-\boldsymbol{\varsigma}) \cdot \mathbf{T} \geq 0 .
$$

Let us introduce the function $\Psi$ by

$$
\Psi=e+\mathbf{T} \cdot \boldsymbol{\Lambda}-T S
$$

By taking the time derivative of this relation and substituting in 2.4, we get the energy equation

$$
\rho v-\rho(\dot{\Psi}+\dot{T} S+T \dot{S}-\dot{\mathbf{T}} \cdot \boldsymbol{\Lambda}-\mathbf{T} \cdot \dot{\boldsymbol{\Lambda}})+\operatorname{div} \mathbf{q}+\boldsymbol{\sigma}: \dot{\varepsilon}=0
$$

Substituting 2.12 into 2.11, we obtain the modified Clausius-Duhem inequality including microtemperatures:

$$
-\rho(\dot{\Psi}+\dot{T} S-\dot{\mathbf{T}} \cdot \boldsymbol{\Lambda})+\boldsymbol{\sigma}: \dot{\varepsilon}+\frac{\mathbf{q}}{T} \cdot \nabla T+\frac{\nabla T}{T} \cdot \mathbf{Q} \cdot \mathbf{T}-\mathbf{Q}: \nabla \mathbf{T}+(\mathbf{q}-\boldsymbol{\varsigma}) \cdot \mathbf{T} \geq 0 .
$$


The mechanical behavior is assumed to be elasto-plastic. Consequently, the total strain tensor $\varepsilon$ can be additively decomposed in its elastic (thermoelastic) part $\varepsilon^{e}$ and plastic part $\varepsilon^{p}$ :

$$
\varepsilon=\varepsilon^{e}+\varepsilon^{p}
$$

Note that the tensors $\varepsilon^{e}$ and $\varepsilon^{p}$ cannot be directly expressed in terms of displacement $\mathbf{u}$, unlike the total strain tensor $\varepsilon$ (see Eq. 2.5)). In the present work, attention is restricted to isotropic hardening in the modeling of the mechanical behavior. Consequently, the plastic flow can be defined by the following form of consistency condition (see Naghdi and Trapp [28]):

$$
f=\left(\sigma_{e q}-\sigma_{s}\right) \leq 0, \quad \dot{\varepsilon}_{e q}^{p} \geq 0, \quad \dot{\varepsilon}_{e q}^{p} f=0, \quad \dot{\varepsilon}_{e q}^{p} \dot{f}=0,
$$

where $\sigma_{e q}$ is the equivalent stress, which is function of the Cauchy stress tensor $\boldsymbol{\sigma}$, while $\sigma_{s}$ is the yield stress, viewed as a measure of isotropic hardening and $\dot{\varepsilon}_{e q}^{p}$ is the equivalent plastic strain rate given by

$$
\boldsymbol{\sigma}: \dot{\varepsilon}^{p}=\sigma_{e q} \dot{\varepsilon}_{e q}^{p}
$$

From 2.16 , one can distinguish the following cases

$$
\left\{\begin{array}{lll}
f<0 & \Rightarrow \dot{\varepsilon}_{e q}^{p}=0 & \Rightarrow \text { elastic loading, } \\
f=0 \text { and } \dot{f}<0 & \Rightarrow \dot{\varepsilon}_{e q}^{p}=0 & \Rightarrow \text { elastic unloading, } \\
f=0 \text { and } \dot{f}=0 & \Rightarrow \dot{\varepsilon}_{e q}^{p} \geq 0 & \Rightarrow \text { plastic loading }
\end{array}\right.
$$

In the present contribution, a thermo-gradient-dependent formulation of plasticity is used to express the yield stress function $\sigma_{s}$. Within this formulation, $\sigma_{s}$ is assumed to depend on the equivalent plastic strain measure $\chi$, its Laplacian $\Delta \chi$, the absolute temperature $T$ and the microtemperatures $\mathbf{T}$ :

$$
\sigma_{s}:=\sigma_{s}(\chi, \Delta \chi, T, \mathbf{T})
$$

The equivalent plastic strain rate $\dot{\chi}$ is related to the plastic strain rate $\dot{\boldsymbol{\varepsilon}}^{p}$ through the normality law:

$$
\dot{\varepsilon}^{p}=\dot{\chi} \frac{\partial f}{\partial \boldsymbol{\sigma}} .
$$

The constitutive variables are functions of the elastic strain tensor $\varepsilon^{e}$, the absolute temperature $T$, the microtemperatures $\mathbf{T}$ and the hardening variables $\chi$ and $\Delta \chi$. Hence, within this thermodynamic framework, the Helmholtz free energy $\Psi$ can be expressed as:

$$
\Psi:=\Psi\left(\varepsilon^{e}, \chi, \Delta \chi, T, \mathbf{T}\right)
$$

According to the expression given above for $\Psi$, the time differentiation of Eq. 2.20 can be expanded in terms of its derivatives with respect to the internal state variables, as follows:

$$
\dot{\Psi}=\frac{\partial \Psi}{\partial \varepsilon^{e}}: \dot{\varepsilon}^{e}+\frac{\partial \Psi}{\partial \chi} \dot{\chi}+\frac{\partial \Psi}{\partial \Delta \chi} \Delta \dot{\chi}+\frac{\partial \Psi}{\partial T} \dot{T}+\frac{\partial \Psi}{\partial \mathbf{T}} \dot{\mathbf{T}} .
$$


By substituting Eq. 2.21 into Eq. 2.14 and using Eq. 2.15, one obtains the following thermodynamic constraint:

$$
\begin{array}{r}
\left(\boldsymbol{\sigma}-\rho \frac{\partial \Psi}{\partial \boldsymbol{\varepsilon}^{e}}\right): \dot{\boldsymbol{\varepsilon}}^{e}+\boldsymbol{\sigma}: \dot{\boldsymbol{\varepsilon}}^{p}-\rho\left(S+\frac{\partial \Psi}{\partial T}\right) \dot{T}+\rho\left(\boldsymbol{\Lambda}-\frac{\partial \Psi}{\partial \mathbf{T}}\right) \cdot \dot{\mathbf{T}}-\rho \frac{\partial \Psi}{\partial \chi} \dot{\chi}-\rho \frac{\partial \Psi}{\partial \Delta \chi} \Delta \dot{\chi} \\
+\frac{\mathbf{q}}{T} \cdot \nabla T+\frac{\nabla T}{T} \cdot \mathbf{Q} \cdot \mathbf{T}-\mathbf{Q}: \nabla \mathbf{T}+(\mathbf{q}-\boldsymbol{\varsigma}) \cdot \mathbf{T} \geq 0
\end{array}
$$

The constitutive equations are compatible with the energy equation if they satisfy the following relations:

$$
\boldsymbol{\sigma}=\rho \frac{\partial \Psi}{\partial \varepsilon^{e}}, \quad S=-\frac{\partial \Psi}{\partial T}, \quad \boldsymbol{\Lambda}=\frac{\partial \Psi}{\partial \mathbf{T}} .
$$

Also, we define the thermodynamic forces $R$ and $R^{g}$ associated with the internal variables $\chi$ and $\Delta \chi$, respectively, by the following relations:

$$
R=\rho \frac{\partial \Psi}{\partial \chi}, \quad R^{g}=\rho \frac{\partial \Psi}{\partial \Delta \chi} .
$$

The yield stress $\sigma_{s}$ may be expressed in terms of its initial value $\sigma_{y}$ and the thermodynamic forces $R$ and $R^{g}$ by the following relation:

$$
\sigma_{s}=\sigma_{y}+R+R^{g}
$$

The introduction of Eqs. 2.23) and 2.24 reduces Eq. 2.22 to:

$$
\boldsymbol{\sigma}: \dot{\boldsymbol{\varepsilon}}^{p}-R \dot{\chi}-R^{g} \Delta \dot{\chi}+\frac{\mathbf{q}}{T} \cdot \nabla T+\frac{\nabla T}{T} \cdot \mathbf{Q} \cdot \mathbf{T}-\mathbf{Q}: \nabla \mathbf{T}+(\mathbf{q}-\boldsymbol{\varsigma}) \cdot \mathbf{T} \geq 0 .
$$

Using Eq. 2.23 again, $\rho \dot{\Psi}$ may be reduced to the following expression:

$$
\rho \dot{\Psi}=\boldsymbol{\sigma}: \dot{\varepsilon}^{e}-\rho S \dot{T}+\rho \boldsymbol{\Lambda} \cdot \dot{\mathbf{T}}+R \dot{\chi}+R^{g} \Delta \dot{\chi}
$$

Then, the energy equation 2.13 becomes:

$$
\rho v+\boldsymbol{\sigma}: \dot{\boldsymbol{\varepsilon}}^{p}-R \dot{\chi}-R^{g} \Delta \dot{\chi}-\rho T \dot{S}+\rho \mathbf{T} \cdot \dot{\boldsymbol{\Lambda}}+\operatorname{div} \mathbf{q}=0 .
$$

We consider a reference configuration, which is in thermal equilibrium and free from stresses. We expand $\Psi$ as a power series of the independent variables $\varepsilon^{e}, T, \mathbf{T}, \chi$ and $\Delta \chi$, in which only terms of second order or less are kept. Hence, we propose the following free energy function for centrosymmetric and homogeneous materials:

$$
\rho \Psi=\frac{1}{2} \varepsilon^{e}: \mathbf{C}: \varepsilon^{e}-\theta \mathbf{K}: \varepsilon^{e}-\frac{\rho}{2} \frac{C_{v}}{T_{0}} \theta^{2}-\frac{1}{2} \mathbf{T} \cdot \mathbf{B} \cdot \mathbf{T}+\frac{1}{2} h \chi^{2}-\frac{1}{2} h^{g}(\Delta \chi)^{2}-\xi_{\text {iso }} \chi \theta-\xi_{\text {iso }}^{g} \Delta \chi \theta,
$$

where $T_{0}$ is the initial temperature and $\theta$ the difference between the absolute temperature $T$ and $T_{0}$, i.e.,

$$
\theta=T-T_{0}
$$

We assume that $\left|\theta / T_{0}\right|<<1$. $C_{v}$ is the specific heat, $h$ and $h^{g}$ stand for the local hardening/softening modulus and the second-order non-local gradient, respectively. $\xi_{\text {iso }}$ and $\xi_{i s o}^{g}$ are parameters related to the coupling 
between isotropic hardening and temperature effects, $\mathbf{C}$ is the fourth-order elasticity tensor, $\mathbf{K}$ and $\mathbf{B}$ are second-order tensors defined by the following relations:

$$
\mathbf{K}=\alpha \mathbf{C}: \mathbf{I}, \quad \mathbf{B}=\tilde{b} \widetilde{\mathbf{C}}: \mathbf{I},
$$

where $\alpha$ is the thermal expansion parameter, $\tilde{b}$ is the microthermal conductivity parameter, $\widetilde{\mathbf{C}}$ is the fourth-order microtemperatures tensor and $\mathbf{I}$ is the second-order identity tensor.

Making use of the quadratic form 2.29 , we have:

$$
\begin{aligned}
& \rho \frac{\partial^{2} \Psi}{\partial \theta \partial \varepsilon^{e}}=-\mathbf{K}, \quad \rho \frac{\partial^{2} \Psi}{\partial \theta \partial \chi}=-\xi_{\text {iso }}, \quad \rho \frac{\partial^{2} \Psi}{\partial \theta \partial \Delta \chi}=-\xi_{\text {iso }}^{g}, \quad \rho \frac{\partial^{2} \Psi}{\partial \mathbf{T}^{2}}=-\mathbf{B}, \\
& \frac{\partial^{2} \Psi}{\partial \theta^{2}}=-\frac{C_{v}}{T_{0}}, \quad R=\rho \frac{\partial \Psi}{\partial \chi}=h \chi-\xi_{\text {iso }} \theta, \quad R^{g}=\rho \frac{\partial \Psi}{\partial \Delta \chi}=-h^{g} \Delta \chi-\xi_{\text {iso }}^{g} \theta .
\end{aligned}
$$

Using again the expression 2.29 of $\rho \Psi$, the constitutive equations 2.23 take the following form:

$$
\begin{aligned}
& \boldsymbol{\sigma}=\mathbf{C}:\left(\varepsilon^{e}-\alpha \theta \mathbf{I}\right) \\
& \rho S=\mathbf{K}: \varepsilon^{e}+\rho \frac{C_{v}}{T_{0}} \theta+\xi_{\text {iso }} \chi+\xi_{i s o}^{g} \Delta \chi \\
& \rho \boldsymbol{\Lambda}=-\mathbf{B} \cdot \mathbf{T}
\end{aligned}
$$

and residual thermal dissipation:

$$
\frac{\mathbf{q}}{T} \cdot \nabla T+\frac{\nabla T}{T} \cdot \mathbf{Q} \cdot \mathbf{T}-\mathbf{Q}: \nabla \mathbf{T}+(\mathbf{q}-\boldsymbol{\varsigma}) \cdot \mathbf{T} \geq 0 .
$$

By using Eqs. 2.24, 2.25, 2.29 and Eq.2.32, the yield stress $\sigma_{s}$ can be expressed as follows:

$$
\sigma_{s}=\sigma_{y}+h \chi-h^{g} \Delta \chi-\xi \theta
$$

where $\xi=\xi_{i s o}+\xi_{i s o}^{g}$ reflects the thermo-mechanical coupling (i.e., the effect of the variation of temperature $\theta$ on the evolution of the yield stress $\sigma_{s}$ ). The material length scale is taken into account in the present model and the corresponding parameter is dependent on the ratio $h^{g} / h$.

By using Eq. (2.23) 2 and (2.30), the energy balance equation 2.28 can be rewritten as:

$$
-\rho\left(\theta+T_{0}\right) \frac{d}{d t}\left(\frac{\partial \Psi}{\partial \theta}\right)=\rho v+\boldsymbol{\sigma}: \dot{\boldsymbol{\varepsilon}}^{p}-R \dot{\chi}-R^{g} \Delta \dot{\chi}+\rho \mathbf{T} \cdot \frac{d}{d t}\left(\frac{\partial \Psi}{\partial \mathbf{T}}\right)+\operatorname{div} \mathbf{q} .
$$

From 2.23$]_{3}$ and 2.33$]_{3}$, we infer that the left-hand side term in the above equation can be expanded by the chain rule as:

$$
\begin{array}{r}
-\rho\left(\theta+T_{0}\right)\left(\frac{\partial^{2} \Psi}{\partial \theta \partial \varepsilon^{e}}: \dot{\varepsilon}^{e}+\frac{\partial^{2} \Psi}{\partial \theta \partial \chi} \dot{\chi}+\frac{\partial^{2} \Psi}{\partial \theta \partial \Delta \chi} \Delta \dot{\chi}+\frac{\partial^{2} \Psi}{\partial \theta^{2}} \dot{\theta}+\frac{\partial^{2} \Psi}{\partial \theta \partial \mathbf{T}} \dot{\mathbf{T}}\right)=\rho v+\boldsymbol{\sigma}: \dot{\varepsilon}^{p} \\
-R \dot{\chi}-R^{g} \Delta \dot{\chi}-\mathbf{T} \cdot \mathbf{B} \cdot \dot{\mathbf{T}}+\operatorname{div} \mathbf{q}
\end{array}
$$

By using Eqs. 2.32, Eq. 2.37) reduces to:

$$
\begin{array}{r}
\left(\theta+T_{0}\right)\left(\mathbf{K}: \dot{\varepsilon}^{e}+\frac{\rho C_{v}}{T_{0}} \dot{\theta}+\xi_{\text {iso }} \dot{\chi}+\xi_{\text {iso }}^{g} \Delta \dot{\chi}\right)=\rho v+\boldsymbol{\sigma}: \dot{\boldsymbol{\varepsilon}}^{p} \\
-h \chi \dot{\chi}+\xi_{\text {iso }} \theta \dot{\chi}+h^{g} \Delta \chi \Delta \dot{\chi}+\xi_{i s o}^{g} \theta \Delta \dot{\chi}-\mathbf{T} \cdot \mathbf{B} \cdot \dot{\mathbf{T}}+\operatorname{div} \mathbf{q} .
\end{array}
$$


The studied material is assumed to be plastically incompressible and, hence, the trace of the plastic strain rate $\dot{\boldsymbol{\varepsilon}}^{p}$ is equal to 0 . Consequently, the double contraction product $\mathbf{K}: \dot{\varepsilon}^{p}$ is equal to 0 and the term $\mathbf{K}: \dot{\varepsilon}^{e}$ may be replaced by $\mathbf{K}: \dot{\varepsilon}$. Thus, Eq. 2.38 becomes

$$
\begin{array}{r}
\left(\theta+T_{0}\right)\left(\mathbf{K}: \dot{\varepsilon}+\frac{\rho C_{v}}{T_{0}} \dot{\theta}+\xi_{\text {iso }} \dot{\chi}+\xi_{i s o}^{g} \Delta \dot{\chi}\right)=\rho v+\boldsymbol{\sigma}: \dot{\varepsilon}^{p} \\
-h \chi \dot{\chi}+\xi_{\text {iso }} \theta \dot{\chi}+h^{g} \Delta \chi \Delta \dot{\chi}+\xi_{\text {iso }}^{g} \theta \Delta \dot{\chi}-\mathbf{T} \cdot \mathbf{B} \cdot \dot{\mathbf{T}}+\operatorname{div} \mathbf{q}
\end{array}
$$

From 2.33$]_{3}$, the balance of the first moment of energy equation 2.6 becomes

$$
-\mathbf{B} \cdot \dot{\mathbf{T}}=\operatorname{div}(\mathbf{Q})+\mathbf{q}-\boldsymbol{\varsigma}+\rho \boldsymbol{\mu} .
$$

In summary, the thermomechanical problem under study is defined by the strain-displacement relationship 2.5), the equilibrium equation 2.3$)_{1}$, the traction boundary condition 2.3$)_{2}$, the consistency condition 2.17$)$, the normality law 2.19), the stress-strain relationship 2.33 1 , the heat equation 2.39) and the first moment of energy equation 2.40 .

It is worth noting that in the context of linear theory, the heat equation 2.39 reduces to

$$
T_{0}\left(\mathbf{K}: \dot{\varepsilon}+\frac{\rho C_{v}}{T_{0}} \dot{\theta}+\xi_{\text {iso }} \dot{\chi}+\xi_{i s o}^{g} \Delta \dot{\chi}\right)=\rho v+\operatorname{div} \mathbf{q},
$$

and the linear version of the residual thermal dissipation:

$$
\mathbf{q} \cdot \nabla T-T_{0} \mathbf{Q}: \nabla \mathbf{T}+T_{0}(\mathbf{q}-\boldsymbol{\varsigma}) \cdot \mathbf{T} \geq 0
$$

The linear approximations for the generalized heat fluxes are given by:

$$
\mathbf{q}=\mathbf{k} \cdot \nabla T+\mathbf{k}_{1} \cdot \mathbf{T}, \quad \mathbf{Q}=-\aleph: \nabla \mathbf{T}, \quad \varsigma=\left(\mathbf{k}-\mathbf{k}_{3}\right) \cdot \nabla T+\left(\mathbf{k}_{1}-\mathbf{k}_{2}\right) \cdot \mathbf{T}
$$

with the constitutive coefficients $\mathbf{k}, \mathbf{k}_{1}, \mathbf{k}_{2}, \mathbf{k}_{3}$ and $\aleph$ satisfy the inequality equation 2.42.

\section{Application to the one-dimensional problem}

To demonstrate the salient feature of the gradient-dependent model of plasticity with microtemperatures, we consider the one-dimensional counterpart of the thermoplasticity model detailed in Section 2. All tensor and vector variables, such as $\varepsilon, \boldsymbol{\sigma}, \mathbf{u}, \mathbf{q}$ and $\mathbf{T}$ become scalars in one-dimensional setting in the form $\varepsilon, \sigma, u, q$ and $\tau$, respectively. Consequently, the equations governing the thermomechanical problem reduce to the following scalar equations:

1. The strain-displacement relationship:

$$
\varepsilon=\frac{\partial u}{\partial x}=u_{x}
$$

which is equivalent to

$$
\dot{\varepsilon}=\frac{\partial \dot{u}}{\partial x}=\dot{u}_{x}
$$


2. The equilibrium equation in the absence of external and body forces:

$$
\sigma_{x}=\rho \ddot{u} .
$$

3. The consistency condition:

$$
\begin{cases}f<0 & \Rightarrow \dot{\chi}=0 \Rightarrow \text { elastic loading, } \\ f=0 \text { and } \dot{f}<0 & \Rightarrow \dot{\chi}=0 \Rightarrow \text { elastic unloading, } \\ f=0 \text { and } \dot{f}=0 & \Rightarrow \dot{\chi} \geq 0 \Rightarrow \text { plastic loading }\end{cases}
$$

where $f=|\sigma|-\sigma_{s}$.

4. The stress-strain relationship:

$$
\sigma=E\left(\varepsilon-\varepsilon^{p}-\alpha \theta\right)
$$

where $E$ is the Young modulus. Elasticity and thermal expansion are assumed to be linear during the loading. Consequently, the scalars $E$ and $\alpha$ remain constant.

5. The linear heat equation:

$$
\alpha E \dot{\varepsilon}+\frac{\rho C_{v}}{T_{0}} \dot{\theta}+\xi_{\text {iso }} \dot{\chi}+\xi_{\text {iso }}^{g} \dot{\chi}_{x x}=\frac{1}{T_{0}} q_{x}
$$

where we have neglected the external heat sources (i.e., $\rho v=0$ ) to simplify the thermomechanical model.

6. The balance of the first moment of energy equation

$$
-\check{b} \dot{\tau}=Q_{x}+q-\varsigma
$$

where we have neglected the first moment of the heat source (i.e., $\mu=0$ ).

The linear approximations for the equations 2.33$]_{3}$ and 2.43 become in this context

$$
\begin{aligned}
\Lambda & =-\check{b} \tau, \\
q & =k \theta_{x}+k_{1} \tau, \\
\varsigma & =\left(k-k_{3}\right) \theta_{x}+\left(k_{1}-k_{2}\right) \tau, \\
Q & =-\left(k_{4}+k_{5}+k_{6}\right) \tau_{x},
\end{aligned}
$$

and the Clausius-Duhem inequality 2.42 becomes

$$
k \geq 0, \quad 3 k_{4}+k_{5}+k_{6} \geq 0, \quad k_{5}+k_{6} \geq 0, \quad k_{6}-k_{5} \geq 0, \quad \frac{4}{T_{0}} k k_{2}-\left(\frac{1}{T_{0}} k_{1}+k_{3}\right)^{2} \geq 0 .
$$

Substituting (3.8) into (3.7), we get the microtemperatures equation:

$$
\check{b} \dot{\tau}-\left(k_{4}+k_{5}+k_{6}\right) \tau_{x x}+k_{2} \tau+k_{3} \theta_{x}=0 .
$$

In the following developments, we will distinguish two cases: elastic loading $(\dot{\chi}=0)$ and elastoplastic loading $(\dot{\chi}>0)$. 


\subsection{Elastic loading}

In this case, $\dot{\chi}=0$, by substituting $(3.8)_{2}$ into $(3.6)$, we obtain the heat equation:

$$
\alpha E \dot{u}_{x}+\frac{\rho C_{v}}{T_{0}} \dot{\theta}-\frac{k}{T_{0}} \theta_{x x}-\frac{k_{1}}{T_{0}} \tau_{x}=0 .
$$

If $\dot{\chi}=0$, the time derivative of the stress-strain relationship (3.5) becomes:

$$
\dot{\sigma}=E(\dot{\varepsilon}-\alpha \dot{\theta})
$$

Then, Eq. 3.12 can be equivalently written as:

$$
\sigma=E(\varepsilon-\alpha \theta)
$$

The insertion of $(3.13)$ into the equilibrium equation (3.3) gives together with $(3.10)$ and (3.11) the linear governing equations of thermoelastic flow with microtemperatures:

$$
\begin{aligned}
\rho \ddot{u}-E u_{x x}+E \alpha \theta_{x} & =0, \\
\alpha E \dot{u}_{x}+\frac{\rho C_{v}}{T_{0}} \dot{\theta}-\frac{k}{T_{0}} \theta_{x x}-\frac{k_{1}}{T_{0}} \tau_{x} & =0, \\
\check{b} \dot{\tau}-\left(k_{4}+k_{5}+k_{6}\right) \tau_{x x}+k_{2} \tau+k_{3} \theta_{x} & =0 .
\end{aligned}
$$

\subsection{Elastoplastic loading}

To Eqs. 3.1 - 3.7), we add the normality law in the one-dimensional case, expressed as follows:

$$
\dot{\varepsilon}^{p}=\dot{\chi} \frac{\partial f}{\partial \sigma}=r \dot{\chi}
$$

where $r$ is the loading indicator, which is equal to 1 (resp. -1) in the case of tension (resp. compression) elastoplastic loading. By introducing the loading indicator $r$, the yield function $f=|\sigma|-\sigma_{s}$ can be equivalently written in this elastoplastic case as:

$$
f=r \sigma-\sigma_{s}
$$

Substituting Eq. 2.35) and (3.5) into (3.16), we get

$$
E\left(\varepsilon-\varepsilon^{p}-\alpha \theta\right)-r \sigma_{y}-h \varepsilon^{p}+h^{g} \varepsilon_{x x}^{p}+\xi r \theta=0 .
$$

By combining Eqs. (3.1) and (3.5), we can easily obtain the expression of the plastic strain $\varepsilon^{p}$ :

$$
\varepsilon^{p}=u_{x}-\frac{1}{E} \sigma-\alpha \theta
$$

Substituting Eq. (3.18) into Eq. (3.17) and using Eq. (3.3), we get:

$$
(E+h) \sigma=E h u_{x}-E h^{g} u_{x x x}+\rho h^{g} \ddot{u}_{x}+\alpha E h^{g} \theta_{x x}-E(h \alpha+\xi r) \theta+E r \sigma_{y} .
$$

Now, inserting Eq. (3.19) into Eq. 3.3, we obtain the equation of motion of a thermo-gradient-dependent strain-softening material in one dimensional setting:

$$
\varrho \ddot{u}=h u_{x x}-h^{g} u_{x x x x}+\frac{\rho h^{g}}{E} \ddot{u}_{x x}+\alpha h^{g} \theta_{x x x}-(h \alpha+\xi r) \theta_{x},
$$


where $\varrho=\frac{\rho(E+h)}{E}$. By using Eqs. (3.2) and (3.15), the heat equation 3.6 can be expressed as:

$$
\alpha E \dot{u}_{x}+\frac{\rho C_{v}}{T_{0}} \dot{\theta}+\frac{1}{r} \xi_{i s o} \dot{\varepsilon}^{p}+\frac{1}{r} \xi_{i s o}^{g} \dot{\varepsilon}_{x x}^{p}=\frac{1}{T_{0}} q_{x} .
$$

Using the time derivative of 3.18 and limiting ourselves to the second order partial derivatives of $u$ and of $\theta$, Eq. (3.21) becomes inserting $(3.8)_{2}$

$$
E\left(\alpha+\frac{\xi_{\text {iso }}}{r(E+h)}\right) \dot{u}_{x}+\left(\frac{\rho C_{v}}{T_{0}}+\frac{\xi_{\text {iso }}(r \xi-E \alpha)}{r(E+h)}\right) \dot{\theta}-\frac{k}{T_{0}} \theta_{x x}-\frac{k_{1}}{T_{0}} \tau_{x}=0 .
$$

Eqs. (3.10) together with 3.20 and 3.22 , give the linear governing equations of thermo-elastoplastic flow with microtemperatures:

$$
\begin{array}{r}
\varrho \ddot{u}-h u_{x x}+h^{g} u_{x x x x}-\frac{\rho h^{g}}{E} \ddot{u}_{x x}-\alpha h^{g} \theta_{x x x}+(h \alpha+\xi r) \theta_{x}=0, \\
E\left(\alpha+\frac{\xi_{\text {iso }}}{r(E+h)}\right) \dot{u}_{x}+\left(\frac{\rho C_{v}}{T_{0}}+\frac{\xi_{\text {iso }}(r \xi-E \alpha)}{r(E+h)}\right) \dot{\theta}-\frac{k}{T_{0}} \theta_{x x}-\frac{k_{1}}{T_{0}} \tau_{x}=0, \\
\check{b} \dot{\tau}-\left(k_{4}+k_{5}+k_{6}\right) \tau_{x x}+k_{2} \tau+k_{3} \theta_{x}=0 .
\end{array}
$$

To avoid that the coupled thermomechanical equations (3.23) be an ill-posed problem, one must ensure that the coefficient $\frac{\rho C_{v}}{T_{0}}+\frac{\xi_{\text {iso }}(r \xi-\alpha E)}{r(E+h)}$ be positive. For this, the condition $\frac{\rho C_{v}}{T_{0}}+\frac{\xi_{\text {iso }} \xi}{E+h}>\frac{E \xi_{\text {iso }} \alpha}{r(E+h)}$ or just $\xi>\frac{\alpha E}{r}$ must be set.

To the field of equations (3.23) or (3.14), we add the appropriate boundary and initial conditions.

\section{Well-posedness of the elastoplastic flow problem}

In this section, we shall study the well-posedness of the system (3.23). Without loss of generality and following [6], we assume that the coefficient $\frac{\rho C_{v}}{T_{0}}+\frac{\xi_{\text {iso }}(r \xi-\alpha E)}{r(E+h)}$ is positive and the term $\alpha h^{g} \theta_{x x x}$ in $3.23 \beta_{1}$ is negligible to make the calculations easier. Then, we consider the following system:

$$
\begin{aligned}
\varrho \ddot{u}(x, t)-\varpi \ddot{u}_{x x}(x, t)+h^{g} u_{x x x x}(x, t)-h u_{x x}(x, t)+\beta \theta_{x}(x, t) & =0, \\
c \dot{\theta}(x, t)+\beta \dot{u}_{x}(x, t)-\kappa \theta_{x x}(x, t)-\kappa_{1} \tau_{x}(x, t) & =0, \\
b \dot{\tau}(x, t)-\kappa_{7} \tau_{x x}(x, t)+\kappa_{2} \tau(x, t)+\kappa_{3} \theta_{x}(x, t) & =0,
\end{aligned}
$$

where $(x, t) \in(0, \ell) \times \mathbb{R}^{+}$. We study the well-posedness of system 4.1 subject to the initial conditions

$$
u(x, 0)=u^{0}(x), \quad u_{t}(x, 0)=v^{0}(x), \quad \theta(x, 0)=\theta^{0}(x), \quad \tau(x, 0)=\tau^{0}(x), \quad x \in(0, \ell) .
$$

We consider clamped boundary conditions for $u$ and Dirichlet boundary condition for $\theta$ and $\tau$, that is:

$$
u(x, t)=u_{x}(x, t)=\theta(x, t)=\tau(x, t)=0, \quad \text { on } x=0, \ell, \quad t>0 .
$$

The variable $u=u(x, t)$ represents the vertical deflection of the bar of length $\ell$ with respect to its reference configuration, $\theta=\theta(x, t)$ accounting for the variation of temperature with respect to its reference value and 
$\tau=\tau(x, t)$ represents the microtemperatures variable. In 4.1 , the coefficients are given by

$$
\begin{aligned}
& \varrho=\frac{\rho(E+h)}{E}, \quad \beta=h \alpha+\xi r, \quad c=\omega\left(\frac{\rho C_{v}}{T_{0}}+\frac{\xi_{\text {iso }}(r \xi-\alpha E)}{r(E+h)}\right), \\
& \kappa=\frac{k \omega}{T_{0}}, \kappa_{1}=\frac{k_{1} \omega}{T_{0}}, b=\check{b} \omega, \kappa_{i}=k_{i} \omega, \quad i=2, \cdots, 6, \kappa_{7}=\kappa_{4}+\kappa_{5}+\kappa_{6} .
\end{aligned}
$$

Since $\omega>0$, the dissipations inequalities 3.9 become

$$
\kappa \geq 0, \quad 3 \kappa_{4}+\kappa_{5}+\kappa_{6} \geq 0, \quad \kappa_{5}+\kappa_{6} \geq 0, \quad \kappa_{6}-\kappa_{5} \geq 0, \quad 4 \kappa \kappa_{2}-\left(\kappa_{1}+\kappa_{3}\right)^{2} \geq 0 .
$$

Note that the last condition in 4.5 implies that

$$
\kappa\left\|\theta_{x}\right\|^{2}+\kappa_{2}\|\tau\|^{2}+\left(\kappa_{1}+\kappa_{3}\right) \Re e\left(\theta_{x}, \tau\right) \geq 0 .
$$

We now transform the initial-boundary-value problem given by equations (4.1)- 4.3 to an abstract problem on a suitable Hilbert space. Well-posedness is then obtained by using semigroup theory. Putting $\dot{u}=v, 4.1$. becomes

$$
\left(\begin{array}{cccc}
1 & 0 & 0 & 0 \\
0 & \varrho-\varpi D^{2} & 0 & 0 \\
0 & 0 & c & 0 \\
0 & 0 & 0 & b
\end{array}\right) \frac{d}{d t}\left(\begin{array}{c}
u \\
v \\
\theta \\
\tau
\end{array}\right)=\left(\begin{array}{cccc}
0 & 1 & 0 & 0 \\
\left(h-h^{g} D^{2}\right) D^{2} & 0 & -\beta D & 0 \\
0 & -\beta D & \kappa D^{2} & \kappa_{1} D \\
0 & 0 & -\kappa_{3} D & \kappa_{7} D^{2}-\kappa_{2}
\end{array}\right)\left(\begin{array}{c}
u \\
v \\
\theta \\
\tau
\end{array}\right),
$$

where $D^{k}=\frac{\partial^{k}}{\partial x^{k}}$. Set

$$
\mathscr{A}=\left(\begin{array}{cccc}
1 & 0 & 0 & 0 \\
0 & \varrho-\varpi D^{2} & 0 & 0 \\
0 & 0 & c & 0 \\
0 & 0 & 0 & b
\end{array}\right), \quad \mathscr{B}=\left(\begin{array}{cccc}
0 & 1 & 0 & 0 \\
\left(h-h^{g} D^{2}\right) D^{2} & 0 & -\beta D & 0 \\
0 & -\beta D & \kappa D^{2} & \kappa_{1} D \\
0 & 0 & -\kappa_{3} D & \kappa_{7} D^{2}-\kappa_{2}
\end{array}\right) .
$$

Then, Eq. 4.7 can be written into Sobolev equation

$$
\mathscr{A} \frac{d \mathcal{U}}{d t}=\mathscr{B} \mathcal{U}, \quad \mathcal{U}(0)=\mathcal{U}_{0}
$$

where

$$
\mathcal{U}=(u, v, \theta, \tau), \quad \mathcal{U}_{0}=\left(u^{0}, v^{0}, \theta^{0}, \tau^{0}\right) .
$$

Put

$$
\begin{aligned}
\mathscr{H} & =H_{0}^{2}(\Omega) \times L^{2}(\Omega) \times L^{2}(\Omega) \times L^{2}(\Omega), \\
\mathscr{D}(\mathscr{A}) & =H_{0}^{2}(\Omega) \times\left(H^{2}(\Omega) \cap H_{0}^{1}(\Omega)\right) \times L^{2}(\Omega) \times L^{2}(\Omega), \\
\mathscr{D}\left(\mathscr{A}^{1 / 2}\right) & =H_{0}^{2}(\Omega) \times H_{0}^{1}(\Omega) \times L^{2}(\Omega) \times L^{2}(\Omega)=\mathscr{K}, \\
\mathscr{D}(\mathscr{B}) & =\left(H^{4}(\Omega) \cap H_{0}^{2}(\Omega)\right) \times H_{0}^{2}(\Omega) \times\left(H^{2}(\Omega) \cap H_{0}^{1}(\Omega)\right) \times\left(H^{2}(\Omega) \cap H_{0}^{1}(\Omega)\right),
\end{aligned}
$$

where $\Omega=(0, \ell)$ and $\mathscr{H}$ and $\mathscr{K}$ are Hilbert spaces. 
We define the inner products of $\mathscr{H}$ and $\mathscr{K}$ as follows:

$$
\begin{aligned}
& \left(\left(\begin{array}{l}
u \\
v \\
\theta \\
\tau
\end{array}\right),\left(\begin{array}{c}
u^{\prime} \\
v^{\prime} \\
\theta^{\prime} \\
\tau^{\prime}
\end{array}\right)\right)_{\mathscr{H}}=h^{g}\left(D^{2} u, D^{2} u^{\prime}\right)_{L^{2}(\Omega)}+h\left(D u, D u^{\prime}\right)_{L^{2}(\Omega)}+\left(v, v^{\prime}\right)_{L^{2}(\Omega)}+\left(\theta, \theta^{\prime}\right)_{L^{2}(\Omega)}+\left(\tau, \tau^{\prime}\right)_{L^{2}(\Omega)}, \\
& \left(\left(\begin{array}{c}
u \\
v \\
\theta \\
\tau
\end{array}\right),\left(\begin{array}{c}
u^{\prime} \\
v^{\prime} \\
\theta^{\prime} \\
\tau^{\prime}
\end{array}\right)\right)_{\mathscr{K}}=h^{g}\left(D^{2} u, D^{2} u^{\prime}\right)_{L^{2}(\Omega)}+h\left(D u, D u^{\prime}\right)_{L^{2}(\Omega)}+\varpi\left(D v, D v^{\prime}\right)_{L^{2}(\Omega)}+\varrho\left(v, v^{\prime}\right)_{L^{2}(\Omega)}+c\left(\theta, \theta^{\prime}\right)_{L^{2}(\Omega)} \\
& +b\left(\tau, \tau^{\prime}\right)_{L^{2}(\Omega)} .
\end{aligned}
$$

We note that the inner product in $\mathscr{K}$ defines a norm, which is given by

$$
\|\mathcal{U}\|_{\mathscr{K}}^{2}=\int_{0}^{\ell}\left(h^{g}\left|D^{2} u\right|^{2}+h|D u|^{2}+\varpi|D v|^{2}+\varrho|v|^{2}+c|\theta|^{2}+b|\tau|^{2}\right) d x .
$$

This norm is equivalent to the usual norm in $\mathscr{K}$.

Theorem 4.1. The problem (4.9) is well-posed and is governed by a $C_{0}$-contraction semigroup on $\mathscr{K}$.

Proof. According to Goldstein [15] (see Theorem 11.11), it is sufficient to prove that $\mathscr{A}$ is a positive and self-adjoint operator on $\mathscr{H}$, that 0 belongs to the resolvent set of $\mathscr{A}$, i.e., $0 \in \rho(\mathscr{A})$, that $\mathscr{D}(\mathscr{A}) \supset \mathscr{D}(\mathscr{B})$ and that $\mathscr{B}$ is a maximal dissipative operator on $\mathscr{H}$. Since it is easy and classic to prove that $0 \in \rho(\mathscr{A})$ and that $\mathscr{D}(\mathscr{A}) \supset \mathscr{D}(\mathscr{B})$, we will only prove that $\mathscr{A}$ is a positive and self-adjoint operator on $\mathscr{H}$. For all $\mathcal{U}=(u, v, \theta, \tau) \in \mathscr{D}(\mathscr{A})$, we have

$$
\begin{aligned}
(\mathscr{A} \mathcal{U}, \mathcal{U})_{\mathscr{H}} & =\left(\left(\begin{array}{c}
u \\
\left(\varrho-\varpi D^{2}\right) v \\
c \theta \\
b \tau
\end{array}\right),\left(\begin{array}{c}
u \\
v \\
\theta \\
\tau
\end{array}\right)\right)_{\mathscr{H}} \\
& =h^{g}\left\|D^{2} u\right\|_{L^{2}(\Omega)}^{2}+h\|D u\|_{L^{2}(\Omega)}^{2}+\varrho\|v\|_{L^{2}(\Omega)}^{2}+\varpi\|D v\|_{L^{2}(\Omega)}^{2}+c\|\theta\|_{L^{2}(\Omega)}^{2}+b\|\tau\|_{L^{2}(\Omega)}^{2} \geq 0,
\end{aligned}
$$

which shows that $\mathscr{A}$ is a positive operator on $\mathscr{H}$. To obtain

$$
\left(\mathscr{A} \mathcal{U}, \mathcal{U}^{\prime}\right)_{\mathscr{H}}=\left(\mathcal{U}, \mathscr{A} \mathcal{U}^{\prime}\right)_{\mathscr{H}}
$$

we observe that

$$
\begin{aligned}
\left(\left(\varrho-\varpi D^{2}\right) v, v^{\prime}\right) & =\varrho\left(v, v^{\prime}\right)_{L^{2}(\Omega)}-\varpi\left(D^{2} v, v^{\prime}\right)_{L^{2}(\Omega)} \\
& =\varrho\left(v, v^{\prime}\right)_{L^{2}(\Omega)}-\varpi\left(v, D^{2} v^{\prime}\right)_{L^{2}(\Omega)} \\
& =\left(v,\left(\varrho-\varpi D^{2}\right) v^{\prime}\right)_{L^{2}(\Omega)} .
\end{aligned}
$$


Hence the operator $\mathscr{A}$ is self adjoint on $\mathscr{H}$.

To prove that $\mathscr{B}$ is a maximal dissipative operator on $\mathscr{H}$, we have for all $\mathcal{U}=(u, v, \theta, \tau) \in \mathscr{D}(\mathscr{B})$

$$
\begin{aligned}
(\mathscr{B U}, \mathcal{U})_{\mathscr{H}} & =\left(\left(\begin{array}{c}
v \\
\left(h-h^{g} D^{2}\right) D^{2} u-\beta D \theta \\
-\beta D v+\kappa D^{2} \theta+\kappa_{1} D \tau \\
\kappa_{7} D^{2} \tau-\kappa_{2} \tau-\kappa_{3} D \theta
\end{array}\right),\left(\begin{array}{c}
u \\
v \\
\theta \\
\tau
\end{array}\right)\right)_{\mathscr{H}} \\
& =h^{g}\left(D^{2} v, D^{2} u\right)+h(D v, D u)+\left(\left(h-h^{g} D^{2}\right) D^{2} u-\beta D \theta, v\right)+\left(-\beta D v+\kappa D^{2} \theta+\kappa_{1} D \tau, \theta\right) \\
& +\left(\kappa_{7} D^{2} \tau-\kappa_{2} \tau-\kappa_{3} D \theta, \tau\right) \\
& =h^{g}\left(D^{2} v, D^{2} u\right)+h(D v, D u)-h(D u, D v)-h^{g}\left(D^{2} u, D^{2} v\right)-\beta(D \theta, v)+\beta(v, D \theta)-\kappa(D \theta, D \theta) \\
& -\kappa_{1}(\tau, D \theta)-\kappa_{7}(D \tau, D \tau)-\kappa_{2}(\tau, \tau)-\kappa_{3}(D \theta, \tau) \\
& =2 i \Im m\left(h^{g}\left(D^{2} v, D^{2} u\right)+h(D v, D u)+\beta(v, D \theta)\right)-\kappa_{7}\|D \tau\|^{2}-\kappa\|D \theta\|^{2}-\kappa_{2}\|\tau\|^{2} \\
& -\kappa_{1}(\tau, D \theta)-\kappa_{3}(D \theta, \tau)
\end{aligned}
$$

from which it follows that

$$
\Re e(\mathscr{B U}, \mathcal{U})_{\mathscr{H}}=-\kappa_{7}\|D \tau\|^{2}-\kappa\|D \theta\|^{2}-\kappa_{2}\|\tau\|^{2}-\left(\kappa_{1}+\kappa_{3}\right) \Re e(D \theta, \tau)
$$

which proves, from 4.5 (or 4.6$)$ ), that $\mathscr{B}$ is a dissipative operator on $\mathscr{H}$.

To show that Range $(I-\mathscr{B})=\mathscr{H}$, we will prove the existence of a vector of functions $(u, v, \theta, \tau) \in \mathscr{D}(\mathscr{B})$ satisfying

$$
\left(\begin{array}{c}
u \\
v \\
\theta \\
\tau
\end{array}\right)-\mathscr{B}\left(\begin{array}{c}
u \\
v \\
\theta \\
\tau
\end{array}\right)=\left(\begin{array}{c}
f_{1} \\
f_{2} \\
f_{3} \\
f_{4}
\end{array}\right) \in \mathscr{H}
$$

which in terms of the components gives

$$
\begin{aligned}
u-v & =f_{1}, \\
v-\left(h-h^{g} D^{2}\right) D^{2} u+\beta D \theta & =f_{2}, \\
\theta+\beta D v-\kappa D^{2} \theta-\kappa_{1} D \tau & =f_{3}, \\
\tau-\kappa_{7} D^{2} \tau+\kappa_{2} \tau+\kappa_{3} D \theta & =f_{4} .
\end{aligned}
$$

Inserting the first equation into the others, we get

$$
\begin{aligned}
u-\left(h-h^{g} D^{2}\right) D^{2} u+\beta D \theta & =f_{1}+f_{2}, \\
\theta+\beta D u-\kappa D^{2} \theta-\kappa_{1} D \tau & =f_{3}+\beta D f_{1}, \\
\tau-\kappa_{7} D^{2} \tau+\kappa_{2} \tau+\kappa_{3} D \theta & =f_{4}, \\
u=D u=\theta=\tau & =0 \quad \text { on } x=0, \ell .
\end{aligned}
$$


To solve 4.15, we consider the following bilinear form defined on $H_{0}^{2}(\Omega) \times H_{0}^{1}(\Omega) \times H_{0}^{1}(\Omega)$ by

$$
\begin{aligned}
\mathcal{F}\left(\left(\begin{array}{l}
u \\
\theta \\
\tau
\end{array}\right),\left(\begin{array}{l}
\phi \\
\varphi \\
\psi
\end{array}\right)\right) & =(u, \phi)+h(D u, D \phi)+h^{g}\left(D^{2} u, D^{2} \phi\right)+\beta(D \theta, \phi)+(\theta, \varphi)+\kappa(D \theta, D \varphi) \\
& +\beta(D u, \varphi)+\kappa_{1}(\tau, D \varphi)+(\tau, \psi)+\kappa_{7}(D \tau, D \psi)+\kappa_{2}(\tau, \psi)+\kappa_{3}(D \theta, \psi) .
\end{aligned}
$$

We will show that the following variational equation

$$
\mathcal{F}\left(\left(\begin{array}{l}
u \\
\theta \\
\tau
\end{array}\right),\left(\begin{array}{c}
\phi \\
\varphi \\
\psi
\end{array}\right)\right)=\left(f_{1}+f_{2}, \phi\right)+\left(f_{3}+\beta D f_{1}, \varphi\right)+\left(f_{4}, \psi\right),
$$

has a unique solution $(u, \theta, \tau) \in H_{0}^{2}(\Omega) \times H_{0}^{1}(\Omega) \times H_{0}^{1}(\Omega)$ for any $(\phi, \varphi, \psi) \in H_{0}^{2}(\Omega) \times H_{0}^{1}(\Omega) \times H_{0}^{1}(\Omega)$. The bilinear form $\mathcal{F}$ is coercive, because

$$
\begin{aligned}
\mathcal{F}\left(\left(\begin{array}{l}
u \\
\theta \\
\tau
\end{array}\right),\left(\begin{array}{l}
u \\
\theta \\
\tau
\end{array}\right)\right) & =\|u\|^{2}+h\|D u\|^{2}+h^{g}\left\|D^{2} u\right\|^{2}+\beta(D \theta, u)+\|\theta\|^{2}+\kappa\|D \theta\|^{2}-\beta(u, D \theta) \\
& +\kappa_{1}(\tau, D \theta)+\|\tau\|^{2}+\kappa_{7}\|D \tau\|^{2}+\kappa_{2}\|\tau\|^{2}+\kappa_{3}(D \theta, \tau) \\
& \geq C\left(\|u\|^{2}+\|D u\|^{2}+\left\|D^{2} u\right\|^{2}+\|\theta\|^{2}+\|D \theta\|^{2}+\|\tau\|^{2}+\|D \tau\|^{2}\right) .
\end{aligned}
$$

According to Lax-Milgram theorem, there exists a unique solution of (4.16) satisfying

$$
(u, \theta, \tau) \in H_{0}^{2}(\Omega) \times H_{0}^{1}(\Omega) \times H_{0}^{1}(\Omega) .
$$

If we put $\phi=\psi=0$ in 4.16 , then

$$
(\theta, \varphi)+\kappa(D \theta, D \varphi)+\beta(D u, \varphi)-\kappa_{1}(D \tau, \varphi)=\left(f_{3}+\beta D f_{1}, \varphi\right), \quad \forall \varphi \in H_{0}^{1}(\Omega)
$$

which implies that $\theta$ is a weak solution of the equation

$$
\begin{aligned}
\theta-k D^{2} \theta & =f_{3}+\beta D f_{1}-\beta D u+\kappa_{1} D \tau, \quad \text { in } \Omega \\
\theta & =0, \quad \text { at } x=0, \ell .
\end{aligned}
$$

Therefore, $\theta \in H^{2}(\Omega) \cap H_{0}^{1}(\Omega)$. If we put $\varphi=\psi=0$ in 4.16 then

$$
(u, \phi)+h(D u, D \phi)+h^{g}\left(D^{2} u, D^{2} \phi\right)+\beta(D \theta, \phi)=\left(f_{1}+f_{2}, \phi\right), \quad \forall \phi \in H_{0}^{2}(\Omega)
$$

which also implies that $u$ is a weak solution to the problem

$$
\begin{aligned}
u-h D^{2} u+h^{g} D^{4} u & =f_{1}+f_{2}-\beta D \theta, \quad \text { in } \Omega \\
u=D u & =0, \quad \text { at } x=0, \ell .
\end{aligned}
$$

Therefore $u \in H^{4}(\Omega) \cap H_{0}^{2}(\Omega)$. Similarly, one can take $\varphi=\phi=0$ in 4.16 to obtain

$$
\left(1+\kappa_{2}\right)(\tau, \psi)+\kappa_{7}(D \tau, D \psi)+\kappa_{3}(D \theta, \psi)=\left(f_{4}, \psi\right), \quad \forall \psi \in H_{0}^{1}(\Omega)
$$


which implies that $\tau$ is a weak solution of the equation

$$
\begin{aligned}
\left(1+\kappa_{2}\right) \tau-\kappa_{7} D^{2} \tau & =f_{4}-\kappa_{3} D \theta, \quad \text { in } \Omega \\
\tau & =0, \quad \text { at } x=0, \ell .
\end{aligned}
$$

Therefore, $\tau \in H^{2}(\Omega) \cap H_{0}^{1}(\Omega)$.

Combining these facts, we conclude that Range $(I-\mathscr{B})=\mathscr{H}$.

Remark 4.1. From Theorem 4.1, we conclude that the operator $\mathscr{A}^{-1} \mathscr{B}$ is the infinitesimal generator of a $C_{0}$ semigroup of linear contractions over the space $\mathscr{K}$ and $0 \in \rho\left(\mathscr{A}^{-1} \mathscr{B}\right)$. Arguing as in the proof of Lemma 5.1 from $[\underline{6}]$, one can show that $\mathscr{A} \mathscr{B}^{-1}: \mathscr{K} \rightarrow \mathscr{K}$ is compact. As a direct result, the spectrum of $\mathscr{A}^{-1} \mathscr{B}$ consists only of isolated eigenvalues with finite multiplicity, i.e. $\sigma\left(\mathscr{A}^{-1} \mathscr{B}\right)=\sigma_{p}\left(\mathscr{A}^{-1} \mathscr{B}\right)$ and satisfies $\Re e(\lambda) \leq 0$, $\forall \lambda \in \sigma\left(\mathscr{A}^{-1} \mathscr{B}\right)$.

\section{$5 \quad$ Analyticity and exponential stability of solutions}

The objective of this section is to prove that the semigroup $S(t)$ associated with problem 4.1 is not analytic, except for a special case. The exponential stability of the solutions is kept in all cases. To this end, we shall use two results stated in the book of Liu and Zheng that ensure the analyticity and exponential stability of solutions (see [17, 24, 30]):

Theorem 5.1. Let us consider $S(t)=e^{\mathscr{C} t}$ a $C_{0}$-semigroup of contractions generated by the operator $\mathscr{C}$ in the Hilbert space $\mathscr{M}$ with resolvent set $\rho(\mathscr{C})$.

(i) $S(t)$ is analytic if and only if

$$
\rho(\mathscr{C}) \supseteq\{i \alpha ; \alpha \in \mathbb{R}\} \equiv i \mathbb{R}
$$

and

$$
\limsup _{|\alpha| \rightarrow \infty}\left\|\alpha(i \alpha I-\mathscr{C})^{-1}\right\|_{\mathscr{L}(\mathscr{M})}<\infty, \quad \alpha \in \mathbb{R} .
$$

(ii) $S(t)$ is exponentially stable if and only if (5.1) holds and

$$
\limsup _{|\alpha| \rightarrow \infty}\left\|(i \alpha I-\mathscr{C})^{-1}\right\|_{\mathscr{L}(\mathscr{M})}<\infty, \quad \alpha \in \mathbb{R}
$$

where the expression $\|\cdot\|_{\mathscr{L}(\mathscr{M})}$ denotes the norm in the space of continuous linear functions in $\mathscr{M}$.

We consider the resolvent equation

$$
\left(\lambda I-\mathscr{A}^{-1} \mathscr{B}\right) \mathcal{U}=F
$$

or

$$
(\lambda \mathscr{A}-\mathscr{B}) \mathcal{U}=\mathscr{A} F
$$

where $\mathcal{U}=(u, v, \theta, \tau) \in \mathscr{K}$ and $F=\left(f_{1}, f_{2}, f_{3}, f_{4}\right)$. We shall take $\lambda=i \alpha$, with $\alpha \in \mathbb{R}$. Therefore, our equation becomes

$$
i \alpha u-v=f_{1}
$$




$$
\begin{aligned}
i \alpha\left(\varrho-\varpi D^{2}\right) v+h^{g} D^{4} u-h D^{2} u+\beta D \theta & =\left(\varrho-\varpi D^{2}\right) f_{2}, \\
i \alpha c \theta-\kappa D^{2} \theta-\kappa_{1} D \tau+\beta D v & =c f_{3}, \\
i \alpha b \tau-\kappa_{7} D^{2} \tau+\kappa_{2} \tau+\kappa_{3} D \theta & =b f_{4} .
\end{aligned}
$$

For the rest, we need to prove the following

Lemma 5.1. The operator $\mathscr{A}^{-1} \mathscr{B}$ satisfies 5.1 .

Proof. We only need to show that there is no point spectrum on the imaginary axis, i.e., $i \mathbb{R} \cap \sigma_{p}\left(\mathscr{A}^{-1} \mathscr{B}\right)=\emptyset$.

Suppose that there exists $\alpha \in \mathbb{R}, \alpha \neq 0$, such that $i \alpha$ is in the spectrum of $\mathscr{A}^{-1} \mathscr{B}$. Since $\mathscr{A}_{\mathscr{B}}^{-1}$ is compact, then $i \alpha$ must be an eigenvalue of $\mathscr{A}^{-1} \mathscr{B}$. Therefore, there is a vector $\mathcal{U} \in \mathscr{D}\left(\mathscr{A}^{-1} \mathscr{B}\right), \mathcal{U} \neq 0$, such that $(i \alpha \mathscr{A}-\mathscr{B}) \mathcal{U}=0$ in $\mathscr{K}$ or equivalently

$$
\begin{aligned}
i \alpha u-v & =0, \\
i \alpha\left(\varrho-\varpi D^{2}\right) v+h^{g} D^{4} u-h D^{2} u+\beta D \theta & =0, \\
i \alpha c \theta-\kappa D^{2} \theta-\kappa_{1} D \tau+\beta D v & =0, \\
i \alpha b \tau-\kappa_{7} D^{2} \tau+\kappa_{2} \tau+\kappa_{3} D \theta & =0 .
\end{aligned}
$$

Since

$$
<(i \alpha \mathscr{A}-\mathscr{B}) \mathcal{U}, \mathcal{U}>\mathscr{K}=\kappa_{7}\|D \tau\|^{2}+\kappa\|D \theta\|^{2}+\kappa_{2}\|\tau\|^{2}+\left(\kappa_{1}+\kappa_{3}\right) \Re e(D \theta, \tau)=0,
$$

then $\theta=\tau=0$ by 4.5$)_{5}$ and 4.6 . From 5.5$)_{1,3}$, we infer that $u=v=0$. Thus, we have a contradiction and the proof is complete.

\subsection{Lack of analyticity.}

The aim of this subsection is to show that the semigroup $S(t)$ associated with system 4.1 is not analytic in general. To this end, we use the characterization of analytic semigroups given by Theorem 5.1 .

As the method used is not adequate for the boundary conditions 4.3 , we will use for this purpose

$$
u(x, t)=u_{x x}(x, t)=\theta_{x}(x, t)=\tau(x, t)=0, \quad \text { on } \quad x=0, \ell, \quad t>0,
$$

but the result of the analysis also works for boundary conditions 4.3). Under the boundary conditions (5.7), the problem 4.9 is well-posed and is governed by a $C_{0}$-contraction semigroup $S(t)$ on $\mathscr{K}=H^{2}(\Omega) \cap H_{0}^{1}(\Omega) \times$ $H_{0}^{1}(\Omega) \times L_{*}^{2}(\Omega) \times L^{2}(\Omega)$, where $L_{*}^{2}(\Omega)=\left\{w \in L^{2}(\Omega) ; \int_{0}^{\ell} w d x=0\right\}$.

We will show the following theorem.

Theorem 5.2. The semigroup $S(t)$ is not analytic.

Proof. Because of the Theorem 5.1. it is sufficient to show the existence of sequences $\left(\alpha_{n}\right)_{n}$ of real numbers with $\alpha_{n} \rightarrow \infty$ when $n \rightarrow \infty,\left(\mathcal{U}_{n}\right)_{n} \in \mathscr{D}\left(\mathscr{A}^{-1} \mathscr{B}\right)$ and $\left(F_{n}\right)_{n} \in \mathscr{K}$ such that $\left(i \alpha_{n} \mathscr{A}-\mathscr{B}\right) \mathcal{U}_{n}=\mathscr{A} F_{n}$ is bounded in $\mathscr{K}$ and

$$
\lim _{\alpha_{n} \rightarrow \infty}\left\|\alpha_{n} \mathcal{U}_{n}\right\|_{\mathscr{K}}=\infty, \quad \text { as } n \rightarrow \infty
$$


We choose for all $n \in \mathbb{N}, F_{n}=(0, \sin (n x), 0,0)$. Then $F_{n}$ is bounded in $\mathscr{K}$. The solution $\mathcal{U}=\mathcal{U}_{n}=$ $\left(u_{n}, v_{n}, \theta_{n}, \tau_{n}\right)^{T}$ of the equation $\left(i \alpha_{n} \mathscr{A}-\mathscr{B}\right) \mathcal{U}_{n}=\mathscr{A} F_{n}$ satisfies 5.4. On the basis of the boundary conditions 5.7) we can try solutions of the type

$$
u_{n}=A_{n} \sin (n \nu x), \theta_{n}=B_{n} \cos (n \nu x), \tau_{n}=C_{n} \sin (n \nu x),
$$

where $\nu=\frac{\pi}{\ell}, A_{n}, B_{n}$ and $C_{n}$ are unknown functions. Substituting 5.8 into 5.4, we find that $A_{n}, B_{n}$ and $C_{n}$ satisfy

$$
\left\{\begin{array}{l}
\left(-\alpha_{n}^{2} \varrho-\alpha_{n}^{2} \varpi n^{2} \nu^{2}+h n^{2} \nu^{2}+h^{g} n^{4} \nu^{4}\right) A_{n}-\beta n \nu B_{n}=\varrho+\varpi n^{2} \nu^{2}, \\
i \beta \alpha_{n} n \nu A_{n}+\left(i c \alpha_{n}+\kappa n^{2} \nu^{2}\right) B_{n}-\kappa_{1} n \nu C_{n}=0, \\
-\kappa_{3} n \nu B_{n}+\left(i b \alpha_{n}+\kappa_{7} n^{2} \nu^{2}+\kappa_{2}\right) C_{n}=0 .
\end{array}\right.
$$

We choose $\alpha_{n}$ so that $-\alpha_{n}^{2}\left(\varrho+\varpi n^{2} \nu^{2}\right)+h n^{2} \nu^{2}+h^{g} n^{4} \nu^{4}=0$ which implies that $\alpha_{n}= \pm n \nu \sqrt{\frac{h+h^{g} n^{2} \nu^{2}}{\varrho+\varpi n^{2} \nu^{2}}}$. Moreover, the above system leads to

$$
\begin{aligned}
& A_{n}=\frac{\left(i c \alpha_{n}+\kappa n^{2} \nu^{2}\right)\left(\varrho+\varpi n^{2} \nu^{2}\right)}{i \alpha_{n} \beta^{2} n^{2} \nu^{2}}-\frac{\kappa_{1} \kappa_{3}\left(\varrho+\varpi n^{2} \nu^{2}\right) n \nu}{\beta\left(i b \alpha_{n}+\kappa_{7} n^{2} \nu^{2}+\kappa_{2}\right)}, \\
& B_{n}=-\frac{\varrho+\varpi n^{2} \nu^{2}}{\beta n \nu}, \quad C_{n}=-\frac{\kappa_{3}\left(\varrho+\varpi n^{2} \nu^{2}\right)}{\beta\left(i b \alpha_{n}+\kappa_{7} n^{2} \nu^{2}+\kappa_{2}\right)} .
\end{aligned}
$$

The asymptotic behavior of the coefficient $C_{n}$ will give us $C_{n} \sim \frac{\kappa_{3} \varpi}{\kappa_{7} \beta}$ as $n \rightarrow \infty$. This gives $\tau_{n}(x) \sim$ $\frac{\kappa_{3} \varpi}{\kappa_{7} \beta} \sin (n \nu x)$ as $n \rightarrow \infty$. On the other hand, we have

$$
\left\|\mathcal{U}_{n}\right\|_{\mathscr{K}}^{2} \geq b\left\|\tau_{n}\right\|^{2}=\frac{b \kappa_{3} \varpi}{\kappa_{7} \beta} \int_{0}^{\ell}|\sin (n \nu x)|^{2} d x \sim \frac{b \kappa_{3} \varpi \ell}{2 \kappa_{7} \beta} \quad \text { as } n \rightarrow \infty
$$

Thus, we obtain

$$
\lim _{\alpha_{n} \rightarrow \infty}\left\|\alpha_{n} \mathcal{U}_{n}\right\|_{\mathscr{K}} \geq \lim _{\alpha_{n} \rightarrow \infty} \alpha_{n} \frac{b \kappa_{3} \varpi \ell}{2 \kappa_{7} \beta}=\infty
$$

which completes the proof of the theorem.

\subsection{Analyticity for a special case}

In this subsection, we will prove that the semigroup $S(t)$ is analytical for the special case where $h^{g}=0$ and the other coefficients of the system are kept non-zero. In that case, the system remains thermoelastoplastic with a behavior perfectly plastic (without hardening). By using previous arguments, one can check that the corresponding problem remains well-posed and governed by a $C_{0}$-contraction semigroup on $\mathscr{K}$ with compact resolvent operator. The proposed arguments can be adapted for the boundary conditions (5.7). In fact, the result that we show does not depend on the boundary conditions. In view of the assertion $(i)$ of Theorem 5.1 . we prove the following

Lemma 5.2. For any $F \in \mathscr{K}$, there exists a positive constant $C$ such that

$$
\int_{0}^{\ell}\left(|D v|^{2}+|D \theta|^{2}+|D \tau|^{2}+|\tau|^{2}\right) d x \leq C\|F\|_{\mathscr{K}}\|\mathcal{U}\|_{\mathscr{K}} .
$$


Proof. If we multiply the first equation of 5.4 by $-h \overline{D^{2} u}$, the second one by $\bar{v}$, the third one by $\bar{\theta}$ and the last one by $\bar{\tau}$, we obtain after adding the results:

$$
\begin{array}{r}
i \alpha\left(h\|D u\|^{2}+\varrho\|v\|^{2}+\varpi\|D v\|^{2}+c\|\theta\|^{2}+b\|\tau\|^{2}\right)+h \int_{0}^{\ell}(D u \overline{D v}-\overline{D u} D v) d x \\
+\beta \int_{0}^{\ell}(D \theta \bar{v}-\overline{D \theta} v) d x+\int_{0}^{\ell}\left(\kappa_{3} D \theta \bar{\tau}+\kappa_{1} \overline{D \theta} \tau\right) d x+\int_{0}^{\ell}\left(\kappa|D \theta|^{2}+\kappa_{7}|D \tau|^{2}+\kappa_{2}|\tau|^{2}\right) d x=\mathscr{N},
\end{array}
$$

where

$$
\mathscr{N}=h \int_{0}^{\ell} \overline{D u} D f_{1} d x+\varrho \int_{0}^{\ell} \bar{v} f_{2} d x+\varpi \int_{0}^{\ell} \overline{D v} D f_{2} d x+c \int_{0}^{\ell} \bar{\theta} f_{3} d x+b \int_{0}^{\ell} \bar{\tau} f_{4} d x
$$

Remark that $\mathscr{N} \leq C\|F\|_{\mathscr{K}}\|\mathcal{U}\|_{\mathscr{K}}$, for a positive constant $C$. Taking the real part of [5.10), we obtain

$$
\left(\kappa_{3}+\kappa_{1}\right) \Re e \int_{0}^{\ell}(D \theta \bar{\tau}) d x+\int_{0}^{\ell}\left(\kappa|D \theta|^{2}+\kappa_{7}|D \tau|^{2}+\kappa_{2}|\tau|^{2}\right) d x \leq C_{1}\|F\|_{\mathscr{K}}\|\mathcal{U}\|_{\mathscr{K}} .
$$

By using $[4.5]_{5}$ and Poincaré's inequality, there exists a positive constant $C_{1}^{\prime}$ such that,

$$
\int_{0}^{\ell}\left(|D \theta|^{2}+|D \tau|^{2}+|\tau|^{2}\right) d x \leq C_{1}^{\prime}\|F\|_{\mathscr{K}}\|\mathcal{U}\|_{\mathscr{K}}
$$

Multiplying now $(5.4)_{2}$ by $\bar{v}$ we obtain after using $(5.4)_{1}$

$$
i \alpha\left(\varrho \int_{0}^{\ell}|v|^{2} d x+\varpi \int_{0}^{\ell}|D v|^{2} d x+h \int_{0}^{\ell}|D u|^{2} d x\right)=-\beta \int_{0}^{\ell} D \theta \bar{v} d x+\varrho \int_{0}^{\ell} f_{2} \bar{v} d x+\varpi \int_{0}^{\ell} D f_{2} \overline{D v} d x+h \int_{0}^{\ell} D u \overline{D f_{1}} d x .
$$

Taking the imaginary part of 5.12 and using Young's inequality together with 5.11 , we get

$$
\frac{\varrho}{2} \int_{0}^{\ell}|v|^{2} d x+\varpi \int_{0}^{\ell}|D v|^{2} d x+h \int_{0}^{\ell}|D u|^{2} d x \leq C_{2}\|F\|_{\mathscr{K}}\|\mathcal{U}\|_{\mathscr{K}},
$$

for a positive constant $C_{2}$. Then, we deduce that

$$
\int_{0}^{\ell}|D v|^{2} d x \leq C_{2}\|F\|_{\mathscr{K}}\|\mathcal{U}\|_{\mathscr{K}}
$$

Adding (5.14) to 5.11], we get 5.9) with $C=C_{2}+C_{1}^{\prime}$.

Lemma 5.3. For any $F \in \mathscr{K}$, there exists a positive constant $C$ such that

$$
|\alpha|\|\mathcal{U}\|_{\mathscr{K}} \leq C\|F\|_{\mathscr{K}}
$$

where $\mathcal{U}$ is the solution to the problem 4.1. -4.3 .

Proof. We multiply the first equation of (5.4) by $i h \overline{D^{2} u}$, the second by $-i \bar{v}$, the third by $-i \bar{\theta}$, the last one by $-i \bar{\tau}$ and we sum all the results, then we obtain:

$$
\begin{array}{r}
\alpha\left(h\|D u\|^{2}+\varrho\|v\|^{2}+\varpi\|D v\|^{2}+c\|\theta\|^{2}+b\|\tau\|^{2}\right)-i h \int_{0}^{\ell}(D u \overline{D v}-\overline{D u} D v) d x \\
-i \beta \int_{0}^{\ell}(D \theta \bar{v}-\overline{D \theta} v) d x-i \int_{0}^{\ell}\left(\kappa_{3} D \theta \bar{\tau}+\kappa_{1} \overline{D \theta} \tau\right) d x-i \int_{0}^{\ell}\left(\kappa|D \theta|^{2}+\kappa_{7}|D \tau|^{2}+\kappa_{2}|\tau|^{2}\right) d x=-i \mathscr{N} .
\end{array}
$$

Taking the real part, we get after using the norm 4.11

$$
\alpha\|\mathcal{U}\|_{\mathscr{K}}^{2} \leq \Re e\left\{i h \int_{0}^{\ell}(D u \overline{D v}-\overline{D u} D v) d x+i \beta \int_{0}^{\ell}(D \theta \bar{v}-\overline{D \theta} v) d x+i \int_{0}^{\ell}\left(\kappa_{3} D \theta \bar{\tau}+\kappa_{1} \overline{D \theta} \tau\right) d x\right\}
$$




$$
+\quad C_{4}\|F\|_{\mathscr{K}}\|\mathcal{U}\|_{\mathscr{K}}
$$

Using (5.9) and again 4.11, we obtain that

$$
\begin{aligned}
\Re e\left\{i h \int_{0}^{\ell}(D u \overline{D v}-\overline{D u} D v) d x\right\} & \leq 2 h\left(\int_{0}^{\ell}|D v|^{2} d x\right)^{\frac{1}{2}}\left(\int_{0}^{\ell}|D u|^{2} d x\right)^{\frac{1}{2}} \\
& \leq C\left(\|F\|_{\mathscr{K}}\|\mathcal{U}\|_{\mathscr{K}}\right)^{\frac{1}{2}}\left(\int_{0}^{\ell}|D u|^{2} d x\right)^{\frac{1}{2}} \\
& \leq 2 h\left(\|F\|_{\mathscr{K}}\|\mathcal{U}\|_{\mathscr{K}}\right)^{\frac{1}{2}}\|\mathcal{U}\|_{\mathscr{K}}=C\|F\|_{\mathscr{K}}^{\frac{1}{2}}\|\mathcal{U}\|_{\mathscr{K}}^{\frac{3}{2}} .
\end{aligned}
$$

Analogously, we have

$$
\Re e\left\{i \beta \int_{0}^{\ell}(D \theta \bar{v}-\overline{D \theta} v) d x\right\} \leq C\|F\|_{\mathscr{K}}^{\frac{1}{2}}\|\mathcal{U}\|_{\mathscr{K}}^{\frac{3}{2}}
$$

Using again 5.9, we get

$$
\begin{aligned}
\Re e\left\{i \int_{0}^{\ell}\left(\kappa_{3} D \theta \bar{\tau}+\kappa_{1} \overline{D \theta} \tau\right) d x\right\} & \leq\left(\kappa_{1}+\kappa_{3}\right)\left(\int_{0}^{\ell}|D \theta|^{2} d x\right)^{\frac{1}{2}}\left(\int_{0}^{\ell}|\tau|^{2} d x\right)^{\frac{1}{2}} \\
& \leq C\left(\|F\|_{\mathscr{K}}\|\mathcal{U}\|_{\mathscr{K}}\right)^{\frac{1}{2}}\left(\|F\|_{\mathscr{K}}\|\mathcal{U}\|_{\mathscr{K}}\right)^{\frac{1}{2}} \\
& \leq C\|F\|_{\mathscr{K}}\|\mathcal{U}\|_{\mathscr{K}} .
\end{aligned}
$$

Substituting (5.17)-(5.19) into (5.16), we obtain

$$
\alpha\|\mathcal{U}\|_{\mathscr{K}}^{2} \leq C_{5}\|F\|_{\mathscr{K}}^{1 / 2}\|\mathcal{U}\|_{\mathscr{K}}^{3 / 2}+C_{6}\|F\|_{\mathscr{H}}\|\mathcal{U}\|_{\mathscr{H}},
$$

or

$$
\alpha\|\mathcal{U}\|_{\mathscr{K}} \leq C_{5}\|F\|_{\mathscr{K}}^{1 / 2}\|\mathcal{U}\|_{\mathscr{K}}^{1 / 2}+C_{6}\|F\|_{\mathscr{K}}
$$

If we use Young's inequality

$$
C_{5}\|F\|_{\mathscr{K}}^{1 / 2}\|\mathcal{U}\|_{\mathscr{K}}^{1 / 2} \leq \varsigma\|\mathcal{U}\|_{\mathscr{K}}+C_{\varsigma}\|F\|_{\mathscr{K}}
$$

where $\varsigma<\alpha$; then from $(5.20)$ we get

$$
(\alpha-\varsigma)\|\mathcal{U}\|_{\mathscr{K}} \leq C_{7}\|F\|_{\mathscr{K}},
$$

where $C_{8}=C_{\varsigma}+C_{7}$. By choosing $\varsigma=\frac{\alpha}{2}$, we conclude that

$$
\alpha\|\mathcal{U}\|_{\mathscr{K}} \leq C\|F\|_{\mathscr{K}}
$$

which prove the lemma.

Theorem 5.3. The semigroup generated by the operator $\mathscr{A}^{-1} \mathscr{B}$ is analytic.

Proof. Since $\mathscr{A}^{-1} \mathscr{B}$ is the infinitesimal generator of a strongly continuous semigroup, $\mathbb{R}^{+} \subset \rho\left(\mathscr{A}^{-1} \mathscr{B}\right)$ and as $0 \in \rho\left(\mathscr{A}^{-1} \mathscr{B}\right)$ (see remark 4.1 , we conclude that $i \mathbb{R} \subset \rho\left(\mathscr{A}^{-1} \mathscr{B}\right)$. From Lemma 5.3 , we have

$$
\left\|\alpha\left(i \alpha I-\mathscr{A}^{-1} \mathscr{B}\right)^{-1} F\right\|_{\mathscr{K}}=|\alpha|\|U\|_{\mathscr{K}} \leq C\|F\|_{\mathscr{K}} .
$$

Then

$$
\limsup _{|\alpha| \rightarrow \infty}\left\|\alpha\left(i \alpha I-\mathscr{A}^{-1} \mathscr{B}\right)^{-1}\right\|<\infty .
$$


The first consequence of the analyticity result is the exponential decay of solutions.

Corollary 5.1. Let $\mathcal{U}=(u, v, \theta, \tau)$ be a solution to the problem $4.1-4.3$, then $\mathcal{U}$ decays to zero exponentially. That is, there exist two positive constants $M$ and $m$ such that

$$
\|\mathcal{U}(t)\| \leq M \mathcal{U}(0) e^{-m t}, \quad \forall t \geq 0
$$

The second consequence of the analyticity result is the impossibility of localization of solutions in time. That means that the only solution that can be identically zero after a finite period of time is the null solution.

Corollary 5.2. Let $\mathcal{U}=(u, v, \theta, \tau)$ be a solution to the problem 4.1)-(4.3) such that, $u=\theta=\tau \equiv 0$ after a finite time $t_{0}>0$. Then, $u=\theta=\tau \equiv 0$ for every $t \geq 0$.

\subsection{Exponential stability}

We are going to prove that the semigroup $S(t)$ associated with problem $4.1-4.3$ is exponentially stable. Our main result is

Lemma 5.4. The semigroup generated by the operator $\mathscr{A}^{-1} \mathscr{B}$ is exponentially stable.

Proof. In view of the assertion $(i i)$ of Theorem 5.1, it suffices to show that the operator $\mathscr{A}^{-1} \mathscr{B}$ satisfies 5.3 , i.e., there exists a positive constant $C$ such that for $\alpha \in \mathbb{R}$,

$$
\left\|\left(i \alpha I-\mathscr{A}^{-1} \mathscr{B}\right)^{-1} \mathcal{U}\right\|_{\mathscr{K}} \leq C\|F\|_{\mathscr{K}}, \quad \text { for all } F \in \mathscr{K}
$$

Since $C_{0}^{\infty}(\Omega)$ is dense in $\mathscr{K}$, we may assume that $F=\left(f_{1}, f_{2}, f_{3}, f_{4}\right) \in C_{0}^{\infty}(\Omega)$. Put

$$
\left(i \alpha I-\mathscr{A}^{-1} \mathscr{B}\right)^{-1} F=\mathcal{U} \in \mathscr{D}\left(\mathscr{A}^{-1} \mathscr{B}\right)
$$

then

$$
\left(i \alpha I-\mathscr{A}^{-1} \mathscr{B}\right) \mathcal{U}=F
$$

which implies that

$$
(i \alpha \mathscr{A}-\mathscr{B}) \mathcal{U}=\mathscr{A} F \text {. }
$$

To prove 5.21, it is sufficient to show that

$$
\|\mathcal{U}\|_{H^{2}(\Omega) \times\left(H^{1}(\Omega)\right)^{3}} \leq C\|F\|_{\mathscr{K}} .
$$

We can write 5.22 componentwise as (5.4). From Eq. (5.6), we infer that

$$
\Re e<(i \alpha \mathscr{A}-\mathscr{B}) \mathcal{U}, \mathcal{U}>\mathscr{K}=\kappa_{7}\|D \tau\|^{2}+\kappa\|D \theta\|^{2}+\kappa_{2}\|\tau\|^{2}+\left(\kappa_{1}+\kappa_{3}\right) \Re e(D \theta, \tau)
$$

and then from 5.22 , we conclude that

$$
\kappa_{7}\|D \tau\|^{2}+\kappa\|D \theta\|^{2}+\kappa_{2}\|\tau\|^{2}+\left(\kappa_{1}+\kappa_{3}\right) \Re e(D \theta, \tau) \leq C\|F\|_{\mathscr{K}}\|\mathcal{U}\|_{\mathscr{K}},
$$


for a positive constant $C$. Therefore, by Young's inequality, there exists a positive constant $C^{\prime}$ such that

$$
c\|\theta\|^{2}+b\|\tau\|^{2} \leq C^{\prime}\|F\|_{\mathscr{K}}\|\mathcal{U}\|_{\mathscr{K}} .
$$

Multiplying $5.4{ }_{2}$ by $\bar{u}$ and using 5.4$)_{1}$ we obtain

$$
\begin{aligned}
h^{g} \int_{0}^{\ell}\left|D^{2} u\right|^{2} d x+h \int_{0}^{\ell}|D u|^{2} d x & =\varrho \int_{0}^{\ell}|v|^{2} d x+\varpi \int_{0}^{\ell}|D v|^{2} d x+\beta \int_{0}^{\ell} \theta \overline{D u} d x+\varrho \int_{0}^{\ell} f_{2} \bar{u} d x \\
& -\varpi \int_{0}^{\ell} D^{2} f_{2} \bar{u} d x+\varrho \int_{0}^{\ell} v \bar{f}_{1} d x-\varpi \int_{0}^{\ell} v \overline{D^{2} f_{1}} d x,
\end{aligned}
$$

which, using (5.24) and (5.25), implies that

$$
h^{g} \int_{0}^{\ell}\left|D^{2} u\right|^{2} d x+\frac{h}{2} \int_{0}^{\ell}|D u|^{2} d x \leq \varrho \int_{0}^{\ell}|v|^{2} d x+\varpi \int_{0}^{\ell}|D v|^{2} d x+C\|F\| \mathscr{K}\|\mathcal{U}\|_{\mathscr{K}},
$$

for a positive constant $C$.

The next step is to estimate $\int_{0}^{\ell}|v|^{2} d x$ and $\int_{0}^{\ell}|D v|^{2} d x$. To this end, we define the functions $\phi, \omega, \psi$ and $\varphi$ as solutions of the following problems in $[0, \ell]$ :

$$
\begin{aligned}
& D^{2} \phi=-v, D \phi(0)=D \phi(\ell)=0, \quad D^{2} \omega=-\theta, D \omega(0)=D \omega(\ell)=0, \\
& D^{2} \psi=-f_{2}, \psi(0)=\psi(\ell)=0, \quad D^{2} \varphi=-f_{3}, \varphi(0)=\varphi(\ell)=0 .
\end{aligned}
$$

Note that, using Poincaré's inequality, we have

$$
\|\psi\|^{2} \leq C_{p}\left\|f_{2}\right\|^{2}, \quad\|\varphi\|^{2} \leq C_{p}\left\|f_{3}\right\|^{2}
$$

where $C_{p}$ is the Poincaré's constant. Multiplying now $(5.4)_{3}$ by $\overline{D \phi} \in L^{2}(0, \ell)$ and using $(5.4)_{2}$,

$$
i \alpha c \int_{0}^{\ell} \theta \overline{D \phi} d x-\kappa \int_{0}^{\ell} D^{2} \theta \overline{D \phi} d x-\kappa_{1} \int_{0}^{\ell} D \tau \overline{D \phi} d x+\beta \int_{0}^{\ell} D v \overline{D \phi} d x=c \int_{0}^{\ell} f_{3} \overline{D \phi} d x .
$$

Now, we estimate term by term. First, using (5.27), we have

$$
\begin{aligned}
i \alpha c \int_{0}^{\ell} \theta \overline{D \phi} d x & =-i \alpha c \int_{0}^{\ell} D^{2} \omega \overline{D \phi} d x=-i \alpha c \int_{0}^{\ell} D \omega \bar{v} d x \quad\left(\text { use }[5.4)_{1}\right) \\
& =\frac{c h^{g}}{\varrho} \int_{0}^{\ell} D \theta \overline{D^{2} u} d x+\frac{c h}{\varrho} \int_{0}^{\ell} \theta \overline{D u} d x-\frac{c \beta}{\varrho} \int_{0}^{\ell}|\theta|^{2} d x+\frac{c \varpi}{\varrho} \int_{0}^{\ell} D \theta \bar{v} d x \\
& -c \int_{0}^{\ell} \theta \overline{D \psi} d x .
\end{aligned}
$$

Using (5.27) again, we have

$$
\begin{aligned}
c \int_{0}^{\ell} f_{3} \overline{D \phi} d x & =-c \int_{0}^{\ell} D \varphi \bar{v} d x, \quad \beta \int_{0}^{\ell} D v \overline{D \phi} d x=\beta \int_{0}^{\ell}|v|^{2} d x, \\
\kappa \int_{0}^{\ell} D^{2} \theta \overline{D \phi} d x & =\kappa \int_{0}^{\ell} D \theta \bar{v} d x, \quad \kappa_{1} \int_{0}^{\ell} D \tau \overline{D \phi} d x=\kappa_{1} \int_{0}^{\ell} \tau \bar{v} d x .
\end{aligned}
$$

Then, substituting 5.30 and 5.31 into 5.29 results in

$$
\beta \int_{0}^{\ell}|v|^{2} d x=-\frac{c h^{g}}{\varrho} \int_{0}^{\ell} D \theta \overline{D^{2} u} d x-\frac{c h}{\varrho} \int_{0}^{\ell} \theta \overline{D u} d x+\frac{c \beta}{\varrho} \int_{0}^{\ell}|\theta|^{2} d x-\frac{c \varpi}{\varrho} \int_{0}^{\ell} D \theta \bar{v} d x
$$




$$
+c \int_{0}^{\ell} \theta \overline{D \psi} d x+\kappa \int_{0}^{\ell} D \theta \bar{v} d x+\kappa_{1} \int_{0}^{\ell} \tau \bar{v} d x-c \int_{0}^{\ell} D \varphi \bar{v} d x .
$$

Using Sobolev's embedding theorem together with (5.28), we obtain

$$
\frac{5 \varrho}{4} \int_{0}^{\ell}|v|^{2} d x \leq C\|F\|_{\mathscr{K}}\|\mathcal{U}\|_{\mathscr{K}}+\frac{3 h^{g}}{4} \int_{0}^{\ell}\left|D^{2} u\right|^{2} d x+\frac{h}{4} \int_{0}^{\ell}|D u|^{2} d x,
$$

for a positive constant $C$. Multiplying $(5.4)_{3}$ by $\overline{D v}$ we obtain

$$
2 \varpi \int_{0}^{\ell}|D v|^{2} d x \leq C\|F\|_{\mathscr{K}}\|\mathcal{U}\|_{\mathscr{K}} .
$$

Therefore, combining (5.26), (5.33) and (5.34), there exists a constant $C>0$ independent of $\alpha$ and $F \in \mathscr{K}$ such that 5.23 holds, which implies condition 5.3 . Thus, the proof is complete.

\section{$6 \quad$ Numerical experiments}

In this section, we present the numerical approach that we have implemented using the computing environment Matlab (R2015a) to solve the numerical problem associated with Eqs. 4.1)-4.3. Without loss of generality, the numerical approach is developed here for a metal rod, which is considered as one-dimensional (1D) body. To simplify the subsequent numerical developments, the complex consistency condition (3.4) is replaced by an equivalent single equation involving the semismooth Fischer-Burmeister function [13, 14 ]

$$
\Phi=\sqrt{(f)^{2}+\left(\dot{\varepsilon}^{p}\right)^{2}}+f-\left|\dot{\varepsilon}^{p}\right|=0,
$$

with

$$
f=|\sigma|-\sigma_{s}=\operatorname{Er}\left(\varepsilon-\varepsilon^{p}-\alpha \theta\right)-\sigma_{y}-h r \varepsilon^{p}+h^{g} r \varepsilon_{x x}^{p}+\xi \theta .
$$

The use of the Fischer-Burmeister equation instead of the original consistency condition, defined by two inequalities and one equation, allows considerably simplifying the presentation and the numerical approach, as the Fischer-Burmeister equation is always valid independently of the mechanical state (elastic, elastoplastic, tension or compression). Compared to classical models commonly based on elastic or thermoelastic behavior, the consideration of elastoplastic behavior in the present study (with microtemperatures) represents the main originality from both theoretical and numerical points of view.

Analyzing expression (6.2) of function $f$, we deduce that function $\Phi$ only depends on variables $\varepsilon, \varepsilon^{p}, \varepsilon_{x x}^{p}$ and $\theta$ (i.e., independent on variable $\tau$ ). The weak form of Eqs. (3.3) together with 4.1$)_{2,3}$ and 6.1 ) is obtained by setting

$$
\begin{aligned}
\int_{0}^{\ell} \delta u\left(\rho \ddot{u}-\sigma_{x}\right) d x & =0, \\
\int_{0}^{\ell} \delta \theta\left(c \dot{\theta}-\kappa \frac{\partial^{2} \theta}{\partial x^{2}}-\kappa_{1} \frac{\partial \tau}{\partial x}+\beta \frac{\partial \dot{u}}{\partial x}\right) d x & =0, \\
\int_{0}^{\ell} \delta \tau\left(b \dot{\tau}-\kappa_{7} \frac{\partial^{2} \tau}{\partial x^{2}}+\kappa_{2} \tau+\kappa_{3} \frac{\partial \theta}{\partial x}\right) d x & =0, \\
\int_{0}^{\ell} \delta \varepsilon^{p} \Phi d x & =0,
\end{aligned}
$$


where the $\delta$-symbol denotes the variation of the corresponding quantity. Using Eq. 3.5 and the boundary conditions 4.3 and integrating by parts the first three equations of 6.3 , we get

$$
\begin{aligned}
\rho \int_{0}^{\ell} \delta u \ddot{u} d x+E \int_{0}^{\ell} \delta \varepsilon\left(\frac{\partial u}{\partial x}-\varepsilon^{p}-\alpha \theta\right) d x & =0, \\
\int_{0}^{\ell} \kappa\left(\frac{\partial \delta \theta}{\partial x}\right)\left(\frac{\partial \theta}{\partial x}\right) d x+\int_{0}^{\ell} \delta \theta\left(c \dot{\theta}-\kappa_{1} \frac{\partial \tau}{\partial x}+\beta \frac{\partial \dot{u}}{\partial x}\right) d x & =0, \\
\int_{0}^{\ell} \kappa_{7}\left(\frac{\partial \delta \tau}{\partial x}\right)\left(\frac{\partial \tau}{\partial x}\right) d x+\int_{0}^{\ell} \delta \tau\left(b \dot{\tau}+\kappa_{2} \tau+\kappa_{3} \frac{\partial \theta}{\partial x}\right) d x & =0, \\
\int_{0}^{\ell} \delta \varepsilon^{p} \Phi d x & =0 .
\end{aligned}
$$

The main unknowns of the set of equations (6.4) are the displacement $u$, the plastic deformation $\varepsilon^{p}$, its Laplacian $\varepsilon_{x x}^{p}$, the temperature and microtemperatures variations $\theta$ and $\tau$. To determine the distribution and the evolution of these different variables, the studied rod is discretized into $n$ finite elements. Each element is defined by two nodes (one at each end). After a preliminary analysis of the different equations, a linear interpolation is used for fields $u, \theta$, and $\tau$ and a Hermitian interpolation is employed for $\varepsilon^{p}$. Hence, the continuous displacement field $u$, temperature and microtemperatures variations $\theta$ and $\tau$ are defined by the following generic expressions:

$$
u=\mathbf{H} \mathbf{a}, \quad \theta=\mathbf{H} \mathbf{b}, \quad \tau=\mathbf{H} \mathbf{z},
$$

where $\mathbf{H}$ is a matrix containing the linear interpolation polynomials and $\mathbf{a}$ (resp. $\mathbf{b}$ and $\mathbf{z}$ ) is the nodal displacement (resp. temperature and microtemperatures variations) vector defined as follows:

$$
\begin{aligned}
& \mathbf{a}^{T}=<a_{1}, a_{2}, \cdots, a_{n}, a_{n+1}>\text { with } a_{1}=a_{n+1}=0, \\
& \mathbf{b}^{T}=<b_{1}, b_{2}, \cdots, b_{n}, b_{n+1}>\text { with } b_{1}=b_{n+1}=0, \\
& \mathbf{z}^{T}=<z_{1}, z_{2}, \cdots, z_{n}, z_{n+1}>\text { with } z_{1}=z_{n+1}=0 .
\end{aligned}
$$

The strain field $\varepsilon$ is related to the nodal displacement vector a by the following expression:

$$
\varepsilon=\mathbf{H}_{x} \mathbf{a}
$$

In a similar fashion, the plastic deformation $\varepsilon^{p}$ and its Laplacian $\varepsilon_{x x}^{p}$ can be discretized as follows:

$$
\varepsilon^{p}=\mathbf{h}^{T} \mathbf{d}, \quad \varepsilon_{x x}^{p}=\mathbf{h}_{x x}^{T} \mathbf{d},
$$

and $\mathbf{d}$ the nodal plastic strain variation vector defined as follows:

$$
\mathbf{d}^{T}=<d_{1}, d_{2}, \cdots, d_{2 n+1}, d_{2 n+2}>\text { with } d_{2}=d_{2 n+1}=0
$$

To determine the evolution of the different nodal unknowns (namely $\mathbf{a}, \mathbf{b}, \mathbf{z}$ and $\mathbf{d}$ ) during the loading history, the time history is discretized into several time increments, which are typically denoted $I_{\Delta}=[t, t+\Delta t]$. Over each time increment, we assume that $\mathbf{a}(t), \mathbf{b}(t), \mathbf{z}(t)$ and $\mathbf{d}(t)$ are known and the goal is to compute $\Delta \mathbf{a}, \Delta \mathbf{b}$, $\Delta \mathbf{z}$ and $\Delta \mathbf{d}$ over $I_{\Delta}$. To simplify the next development, the different variables will be expressed at $t+\Delta t$ unless 
otherwise stated. By following the same approach developed in [6, 9, Eqs. 6.4 can be transformed into the following matrix form (after some mathematical manipulations):

$$
\begin{aligned}
& \mathbf{K}_{\mathbf{a a}} \Delta \mathbf{a}+\mathbf{K}_{\mathbf{a b}} \Delta \mathbf{b}+\mathbf{K}_{\mathbf{a d}} \Delta \mathbf{d}+\Omega \ddot{\mathbf{a}}=\mathbf{R}_{\mathbf{a}}(t), \\
& \mathbf{K}_{\mathbf{b b}} \Delta \mathbf{b}+\mathbf{K}_{\mathbf{b z}} \Delta \mathbf{z}+\mathbf{C}_{\mathbf{b a}} \dot{\mathbf{a}}+\mathbf{C}_{\mathbf{b b}} \dot{\mathbf{b}}=\mathbf{R}_{\mathbf{b}}(t), \\
& \mathbf{K}_{\mathbf{z b}} \Delta \mathbf{b}+\left(\mathbf{K}_{\mathbf{z z}}+\widetilde{\mathbf{K}}_{\mathbf{z z}} \Delta \mathbf{z}+\mathbf{C}_{\mathbf{z z}} \dot{\mathbf{z}}=\mathbf{R}_{\mathbf{z}}(t),\right. \\
& \mathbf{K}_{\mathbf{d a}} \Delta \mathbf{a}+\mathbf{K}_{\mathbf{d b}} \Delta \mathbf{b}+\mathbf{K}_{\mathbf{d d}} \Delta \mathbf{d}=\mathbf{R}_{\mathbf{d}}(t),
\end{aligned}
$$

where the expressions of the mass matrix $\boldsymbol{\Omega}$, the stiffness matrices $\mathbf{K}_{\mathbf{a a}}, \mathbf{K}_{\mathbf{a b}}, \mathbf{K}_{\mathbf{a d}}, \mathbf{K}_{\mathbf{b b}}, \mathbf{K}_{\mathbf{b z}}, \mathbf{K}_{\mathbf{z b}}, \mathbf{K}_{\mathbf{z z}}$, $\widetilde{\mathbf{K}}_{\mathbf{z z}}, \mathbf{K}_{\mathbf{d a}}, \mathbf{K}_{\mathbf{d b}}, \mathbf{K}_{\mathbf{d d}}$ and the damping matrices $\mathbf{C}_{\mathbf{b a}}, \mathbf{C}_{\mathbf{b b}}, \mathbf{C}_{\mathbf{z z}}$ are given by the following expressions:

$$
\begin{aligned}
& \mathbf{K}_{\mathbf{a a}}=E \int_{0}^{\ell} \mathbf{H}_{x}^{T} \mathbf{H}_{x} d x, \quad \mathbf{K}_{\mathbf{a b}}=-E \alpha \int_{0}^{\ell} \mathbf{H}_{x}^{T} \mathbf{H} d x, \quad \mathbf{K}_{\mathbf{a d}}=-E \int_{0}^{\ell} \mathbf{H}_{x}^{T} \mathbf{h}^{T} d x, \quad \boldsymbol{\Omega}=\rho \int_{0}^{\ell} \mathbf{H}^{T} \mathbf{H} d x, \\
& \mathbf{K}_{\mathbf{b b}}=\int_{0}^{\ell} \kappa \mathbf{H}_{x}^{T} \mathbf{H}_{x} d x, \quad \mathbf{K}_{\mathbf{b z}}=-\int_{0}^{\ell} \kappa_{1} \mathbf{H}^{T} \mathbf{H}_{x} d x, \quad \mathbf{C}_{\mathbf{b a}}=\beta \int_{0}^{\ell} \mathbf{H}^{T} \mathbf{H}_{x} d x, \quad \mathbf{C}_{\mathbf{b b}}=c \int_{0}^{\ell} \mathbf{H}^{T} \mathbf{H} d x, \\
& \mathbf{K}_{\mathbf{z b}}=\int_{0}^{\ell} \kappa_{3} \mathbf{H}^{T} \mathbf{H}_{x} d x, \quad \mathbf{K}_{\mathbf{z z}}=\int_{0}^{\ell} \kappa_{7} \mathbf{H}_{x}^{T} \mathbf{H}_{x} d x, \quad \widetilde{\mathbf{K}}_{\mathbf{z z}}=\int_{0}^{\ell} \kappa_{2} \mathbf{H}^{T} \mathbf{H} d x, \quad \mathbf{C}_{\mathbf{z z}}=b \int_{0}^{\ell} \mathbf{H}^{T} \mathbf{H} d x, \\
& \mathbf{K}_{\mathbf{d a}}=\int_{0}^{\ell} \mathbf{h} \frac{\partial \Phi}{\partial \varepsilon} \mathbf{H}_{x} d x, \quad \mathbf{K}_{\mathbf{d d}}=\int_{0}^{\ell}\left[\mathbf{h} \frac{\partial \Phi}{\partial \varepsilon^{p}} \mathbf{h}^{T}+\mathbf{h} \frac{\partial \Phi}{\partial \varepsilon_{x x}^{p}} \mathbf{h}_{x x}^{T}\right] d x, \quad \mathbf{K}_{\mathbf{d b}}=\int_{0}^{\ell} \mathbf{h} \frac{\partial \Phi}{\partial \theta} \mathbf{H} d x,
\end{aligned}
$$

and the residual vectors $\mathbf{R}_{\mathbf{a}}(t), \mathbf{R}_{\mathbf{b}}(t), \mathbf{R}_{\mathbf{z}}(t), \mathbf{R}_{\mathbf{d}}(t)$ can be easily deduced from the values of the mechanical variables at the beginning of the time increment.

The derivatives $\frac{\partial \Phi}{\partial \varepsilon}, \frac{\partial \Phi}{\partial \theta}, \frac{\partial \Phi}{\partial \varepsilon^{p}}, \frac{\partial \Phi}{\partial \varepsilon_{x x}^{p}}$ can be easily obtained on the basis of Eqs. 6.1] and 6.2):

$$
\begin{aligned}
\frac{\partial \Phi}{\partial \varepsilon} & =\operatorname{Er}\left(1+\frac{f}{\sqrt{f^{2}+\left(\dot{\varepsilon}^{p}\right)^{2}}}\right) \\
\frac{\partial \Phi}{\partial \varepsilon^{p}} & =-h r-E r-\frac{r}{\Delta t}+\frac{\dot{\varepsilon}^{p}-\Delta t(E+h) r f}{\Delta t \sqrt{f^{2}+\left(\dot{\varepsilon}^{p}\right)^{2}}} \\
\frac{\partial \Phi}{\partial \varepsilon_{x x}^{p}} & =h^{g} r\left(1+\frac{f}{\sqrt{f^{2}+\left(\dot{\varepsilon}^{p}\right)^{2}}}\right), \\
\frac{\partial \Phi}{\partial \theta} & =-E r \alpha+\xi-\frac{(E r \alpha-\xi) f}{\sqrt{f^{2}+\left(\dot{\varepsilon}^{p}\right)^{2}}}
\end{aligned}
$$

with $\dot{\varepsilon}^{p}=\frac{\varepsilon^{p}-\varepsilon^{p}(t)}{\Delta t}$. To express system 6.5 in a more compact matrix form, let us introduce the global unknown vector $\mathbf{q}$ defined as follows:

$$
\mathbf{q}=[\dot{\mathbf{a}} \Delta \mathbf{a} \Delta \mathbf{b} \Delta \mathbf{z} \Delta \mathbf{d}] .
$$

Using this new vector, system 6.5 can be recasted in the following form:

$$
\mathbf{A} \dot{\mathbf{q}}+\mathbf{B q}=\mathbf{R}(t)
$$


where matrices $\mathbf{A}, \mathbf{B}$ and vector $\mathbf{R}$ are defined by the following expressions:

$$
\begin{aligned}
& \mathbf{A}=\left(\begin{array}{ccccc}
0 & \Omega & 0 & 0 & 0 \\
\Omega & 0 & 0 & 0 & 0 \\
0 & \mathrm{C}_{\mathrm{ba}} & \mathrm{C}_{\mathrm{bb}} & 0 & 0 \\
0 & 0 & 0 & \mathrm{C}_{\mathrm{zz}} & 0 \\
0 & 0 & 0 & 0 & 0
\end{array}\right), \mathbf{B}=\left(\begin{array}{ccccc}
-\Omega & 0 & 0 & 0 & 0 \\
0 & \mathrm{~K}_{\mathrm{aa}} & \mathbf{K}_{\mathrm{ab}} & 0 & \mathbf{K}_{\mathrm{ad}} \\
0 & 0 & \mathbf{K}_{\mathrm{bb}} & \mathbf{K}_{\mathrm{bz}} & 0 \\
0 & 0 & \mathbf{K}_{\mathrm{zb}} & \mathbf{K}_{\mathrm{zz}}+\widetilde{\mathbf{K}}_{\mathrm{zz}} & 0 \\
0 & \mathrm{~K}_{\mathrm{da}} & \mathbf{K}_{\mathrm{db}} & 0 & \mathbf{K}_{\mathrm{dd}}
\end{array}\right) \\
& \mathbf{R}(t)=\left(\begin{array}{c}
\mathbf{0} \\
\mathbf{R}_{\mathbf{a}}(t) \\
\mathbf{R}_{\mathbf{b}}(t) \\
\mathbf{R}_{\mathbf{z}}(t) \\
\mathbf{R}_{\mathbf{d}}(t)
\end{array}\right) .
\end{aligned}
$$

The first line of Eq. (6.8) (the trivial equation $\boldsymbol{\Omega} \dot{\mathbf{a}}-\boldsymbol{\Omega} \dot{\mathbf{a}}=\mathbf{0}$ ) is added to obtain a first-order form of the numerical system to solve (this allows considerably simplifying the numerical resolution).

By assuming that

$$
\dot{\mathbf{q}}=\frac{\mathbf{q}-\mathbf{q}(t)}{\Delta t},
$$

the unknown vector $\mathbf{q}$ can be deduced from Eq. 6.8:

$$
\mathbf{q}=\left(\frac{\mathbf{A}}{\Delta t}+\mathbf{B}\right)^{-1}\left(\frac{\mathbf{A} \mathbf{q}(t)}{\Delta t}+\mathbf{R}(t)\right)
$$

The system of equations 6.9 is strongly nonlinear, as several components of matrix $\mathbf{B}$ are dependent on the current mechanical state (at the end of the time increment). To solve it, we have used an implicit iterative scheme. At each iteration $k$ of this iterative scheme, the following linear system of algebraic equations should be solved:

$$
\mathbf{q}^{(k+1)}=\left(\frac{\mathbf{A}}{\Delta t}+\mathbf{B}^{(k)}\right)^{-1}\left(\frac{\mathbf{A} \mathbf{q}(t)}{\Delta t}+\mathbf{R}(t)\right)
$$

where

$$
\mathbf{q}^{(0)}=\mathbf{q}(t)
$$

The above linear system has a unique solution, since matrix $\frac{\mathbf{A}}{\Delta t}+\mathbf{B}^{(k)}$ is always invertible. The iterative procedure converges when the following condition is fulfilled:

$$
\left\|\mathbf{q}^{(k+1)}-\mathbf{q}^{(k)}\right\| \leq 10^{-7}
$$

After several numerical simulations, we have observed that the above iterative algorithm converges always in two or three iterations.

We describe in what follows the results of some numerical simulations. The length $\ell$ of the studied rod is set to $1 \mathrm{~m}$. In all simulations, this rod is discretized into 100 finite elements, which have the same initial length $(0.01 \mathrm{~m})$. The adopted time increment $\Delta t$ is set to $10^{-4} \mathrm{~s}$. Some of the selected material parameters correspond 
Table 1: The values of material parameters.

\begin{tabular}{ccc}
\hline$\rho=9010 \mathrm{Kg} / \mathrm{m}^{3}$ & $E=193 \mathrm{GPa}$ & $h=592 \mathrm{MPa}$ \\
$\kappa=386 \mathrm{~W} /(\mathrm{m} \mathrm{K})$ & $h^{g}=-10 \mathrm{MPa} \mathrm{m}^{2}$ & $\alpha=16.6 \times 10^{-16} \mathrm{~K}^{-1}$ \\
$\xi=2 \times 10^{-16} \mathrm{MPaK}$ & $\xi_{i s o}=1 \mathrm{MPaK}$ & $C_{v}=500 \mathrm{~J} / \mathrm{kg} \mathrm{K}$ \\
$T_{0}=298 \mathrm{~K}$ & $\sigma_{y}=20 \mathrm{MPa}$ & $\varpi=4.668 \times 10^{-12} \mathrm{~kg} /(\mathrm{N} \mathrm{m})^{2}$ \\
$\kappa_{1}=\kappa_{3} / 2=2 \times 10^{10} \mathrm{~W} / \mathrm{m}$ & $\kappa_{2}=0.1 \times 10^{10} \mathrm{~W} / \mathrm{m}$ & $b=0.01 \mathrm{~W} . \mathrm{s} / \mathrm{m}$ \\
$\kappa_{4}=0.3 \times 10^{10} \mathrm{~W} / \mathrm{m}$ & $\kappa_{5}=0.5 \times 10^{10} \mathrm{~W} / \mathrm{m}$ & $\kappa_{6}=0.7 \times 10^{10} \mathrm{~W} / \mathrm{m}$ \\
\hline
\end{tabular}

to AK steel 321 material, which are available in [32]. The other parameters are fictive and are defined to clearly emphasize the different numerical phenomena.

The adopted initial conditions, selected to comply with Eqs. (4.2) and (4.3), are provided below:

$$
u_{0}(x)=0, \quad u_{1}(x)=100 x^{2}(\ell-x), \quad \theta_{0}(x)=0, \quad \tau_{0}(x)=0, \quad \text { for all } x \in[0, \ell] .
$$

The evolutions of the components $u, \theta, \tau, \varepsilon^{p}, \varepsilon_{x x}^{p}$ and $\sigma$ for the node located just before the middle of the rod (at $x=0.49 \mathrm{~m}$ ) are given in Figures 1, 3, 5, 7,9 and 11 respectively. These results show that these components strongly oscillate at the beginning of the loading and tend to stabilize at fixed values after few oscillations, which confirms the results obtained in Section 5 about the exponential decay of the solution.

Figures 2, 4, 6, 8, 10 and 12 show, respectively, the spatial distributions of the same components over the rod at $t=10^{-5}, t=5 \times 10^{-4}, t=2 \times 10^{-3}$ and $t=4 \times 10^{-3} \mathrm{~s}$. It is clearly observed in these figures that all the components vanish at the rod boundaries, which is conformal with the boundary conditions prescribed in Eq. (4.3). This result represents a partial validation of the developed numerical tool. Furthermore, the evolution of the distribution of the different variables becomes more and more slow when the time increase (typically for $t \geq 2 \times 10^{-3} s$ ) confirming again the exponential decay of the solution. The result of Figure 2 shows in particular that the displacement $u$ reaches its maximum value at the middle of the rod (at $x=0.5 \mathrm{~m}$ ). At this point, the total deformation $\varepsilon=\frac{\partial u}{\partial x}$ is obviously equal to 0 . Consequently, the components $\theta, \tau, \varepsilon^{p}$, and $\sigma$ are equal to 0 at $x=0.5 \mathrm{~m}$, which can be easily checked in Figs. 4, 6, 8 and 12, respectively.

One can also remark that all theses components have a finite jump at the wavefronts located at $x=0.1 \mathrm{~m}$ and $x=0.9 \mathrm{~m}$. The same situation appears in [23]. This jump, which violates the requirement for continuity, is not physically realistic [2. The behavior shown in Figures 2, 4, 6, 8, 10 and 12 can be described in a similar way. In what follows we will limit ourselves to describing the behavior given by Figure 4 . The temperature rapidly decreases from $x=0 \mathrm{~m}$ until it reaches a peak at the first wavefront located at $x=0.1 \mathrm{~m}$, then increases monotonically to a positive value passing by the middle of the bar where it vanishes. In the positive domain, it reaches a peak value at the second wavefront located at $x=0.9 \mathrm{~m}$ then decreases rapidly to vanish at $x=1 \mathrm{~m}$. 


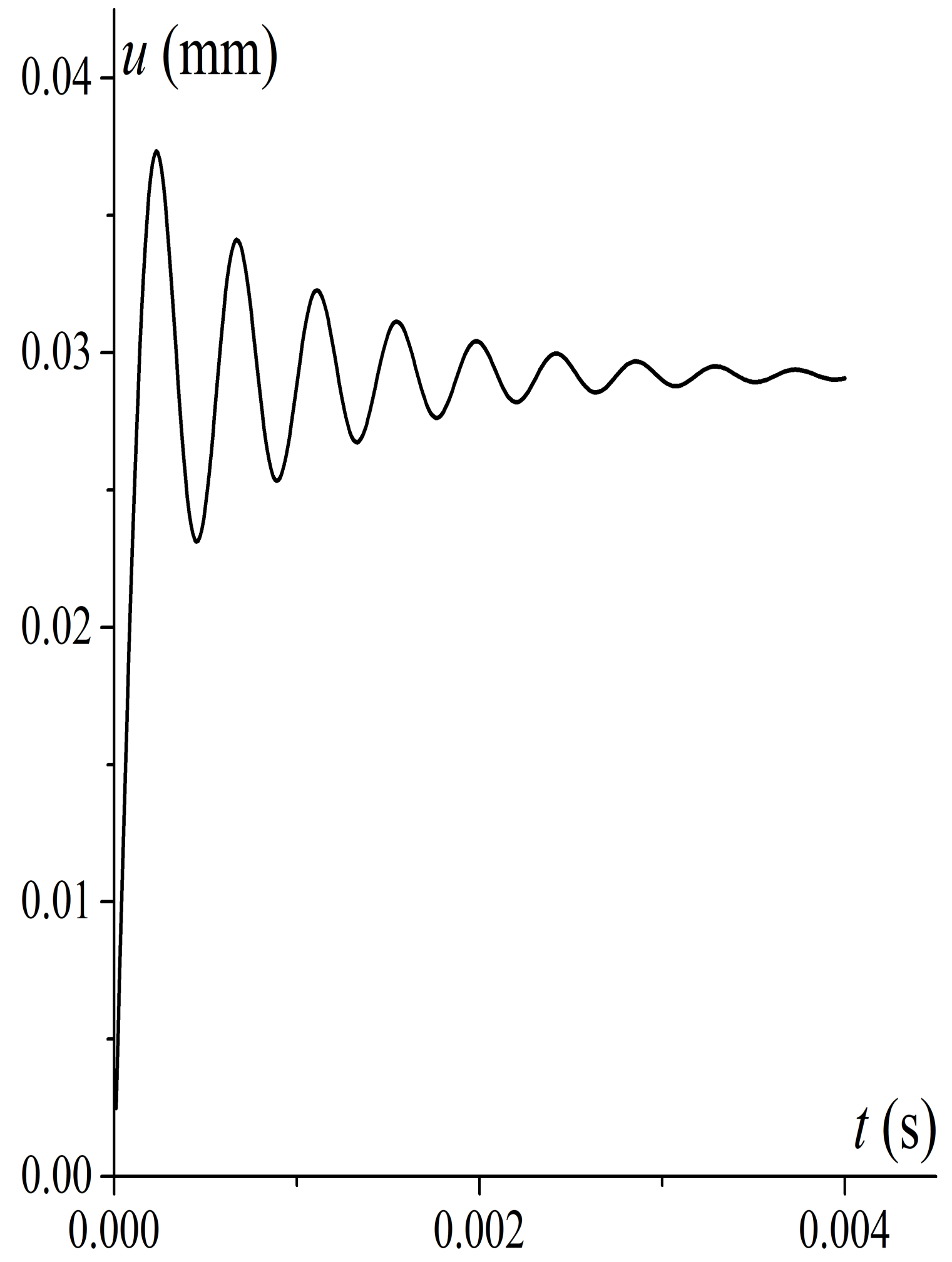

Figure 1: The time evolution of the displacement $u$ at the middle of the bar. 


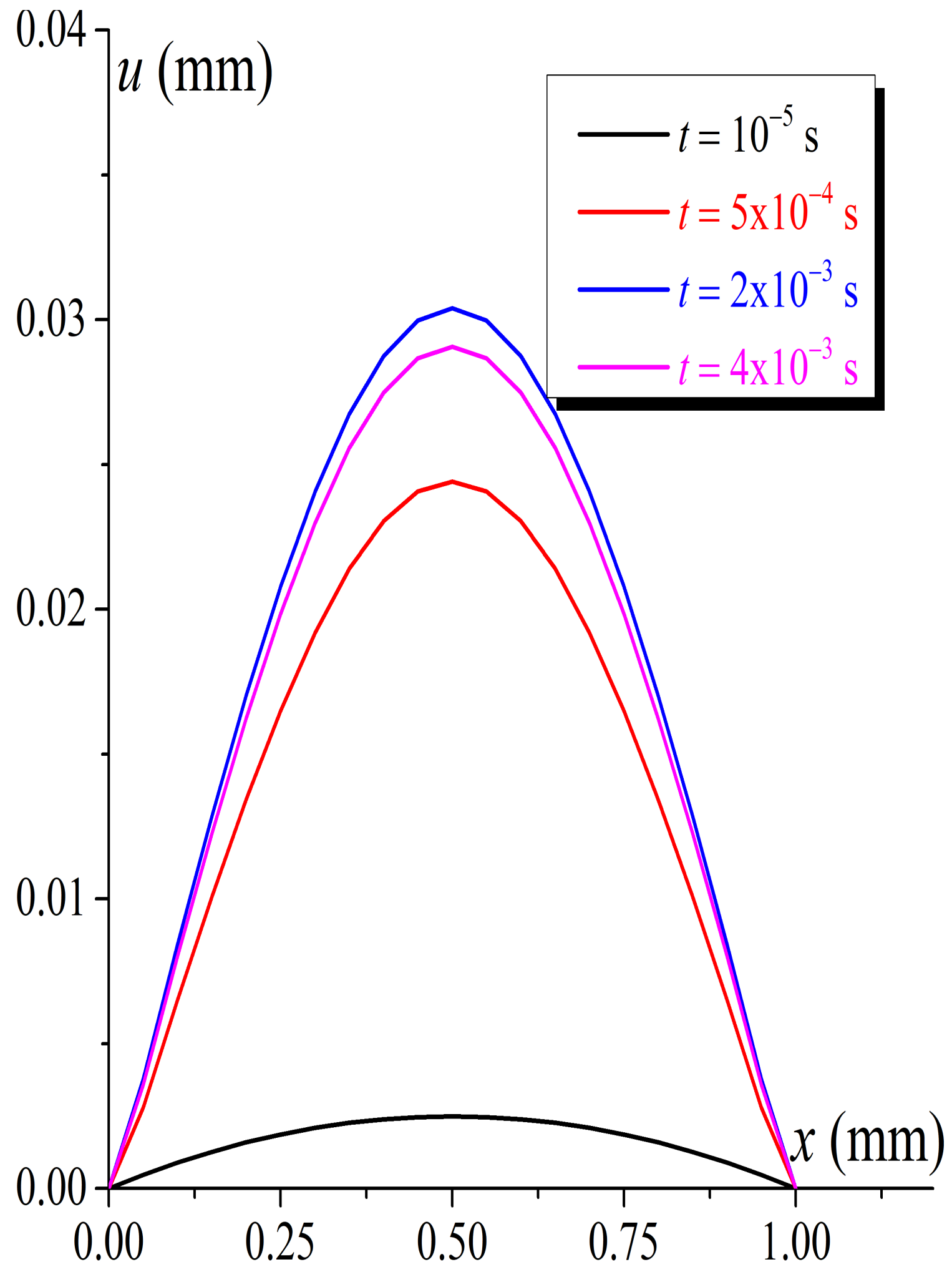

Figure 2: Distribution of the displacement $u$ for different instances. 


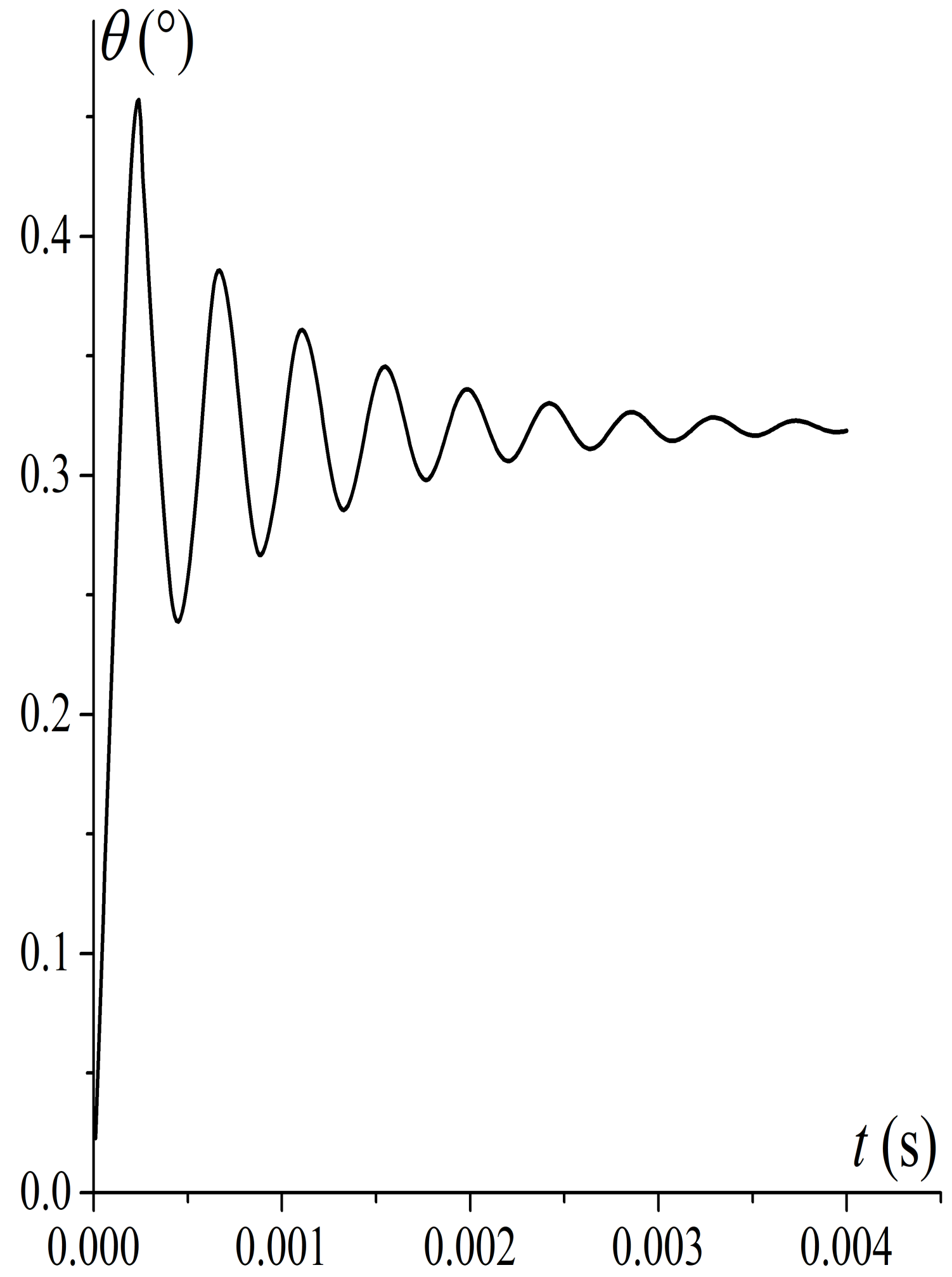

Figure 3: The time evolution of the temperature $\theta$ at the middle of the bar. 


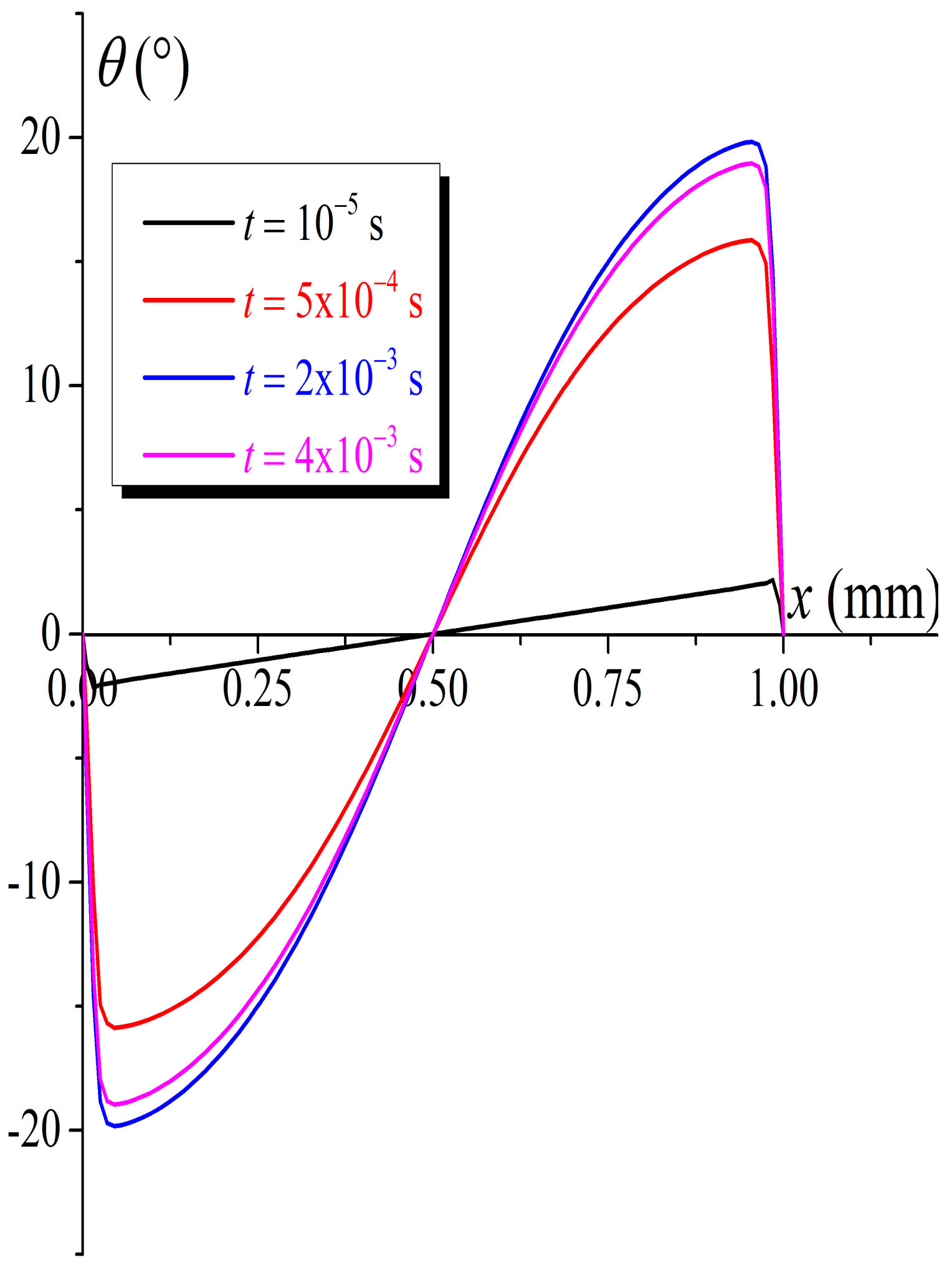

Figure 4: Distribution of the temperature $\theta$ for different instances. 


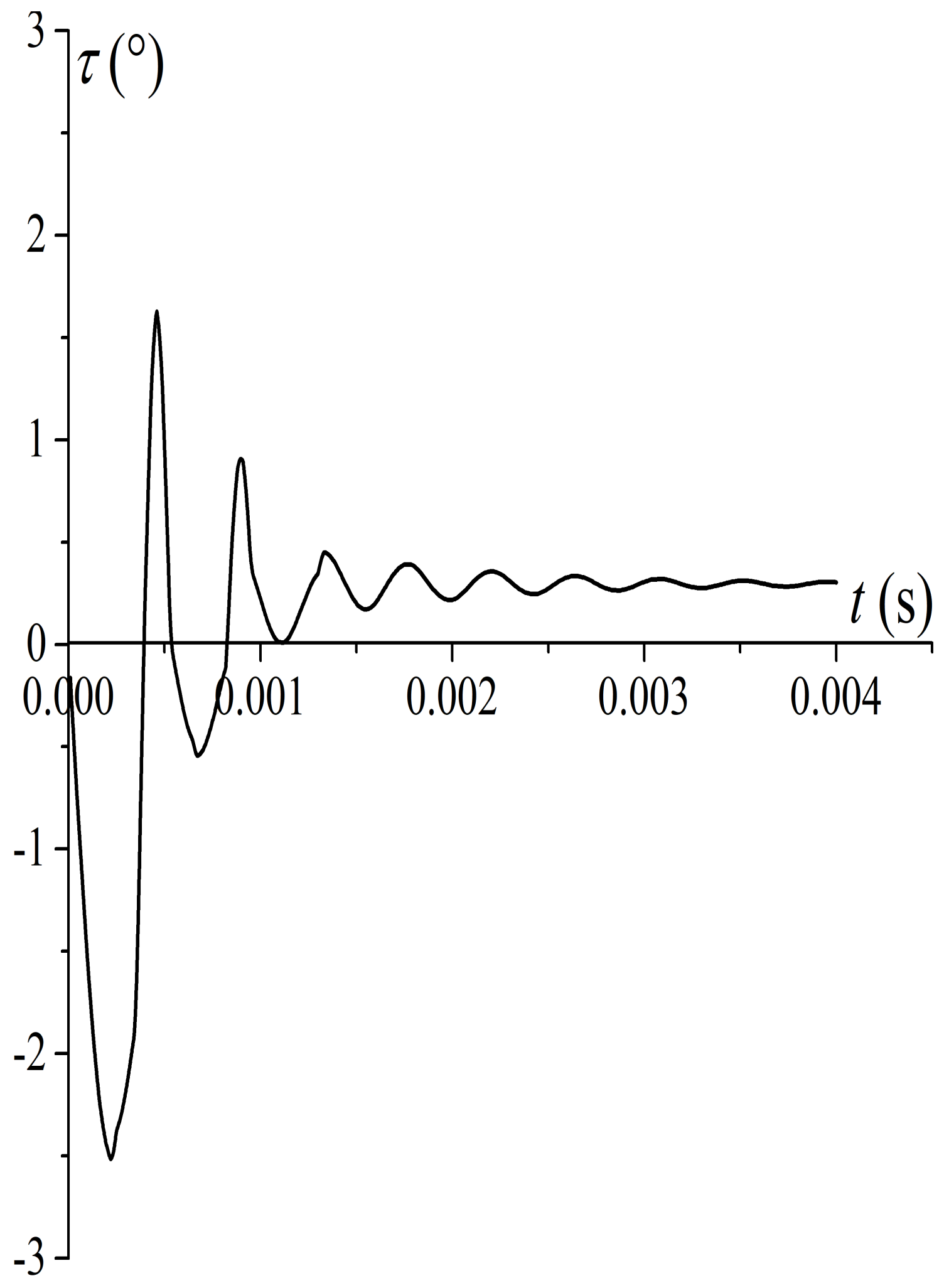

Figure 5: The time evolution of the microtemperatures $\tau$ at the middle of the bar. 


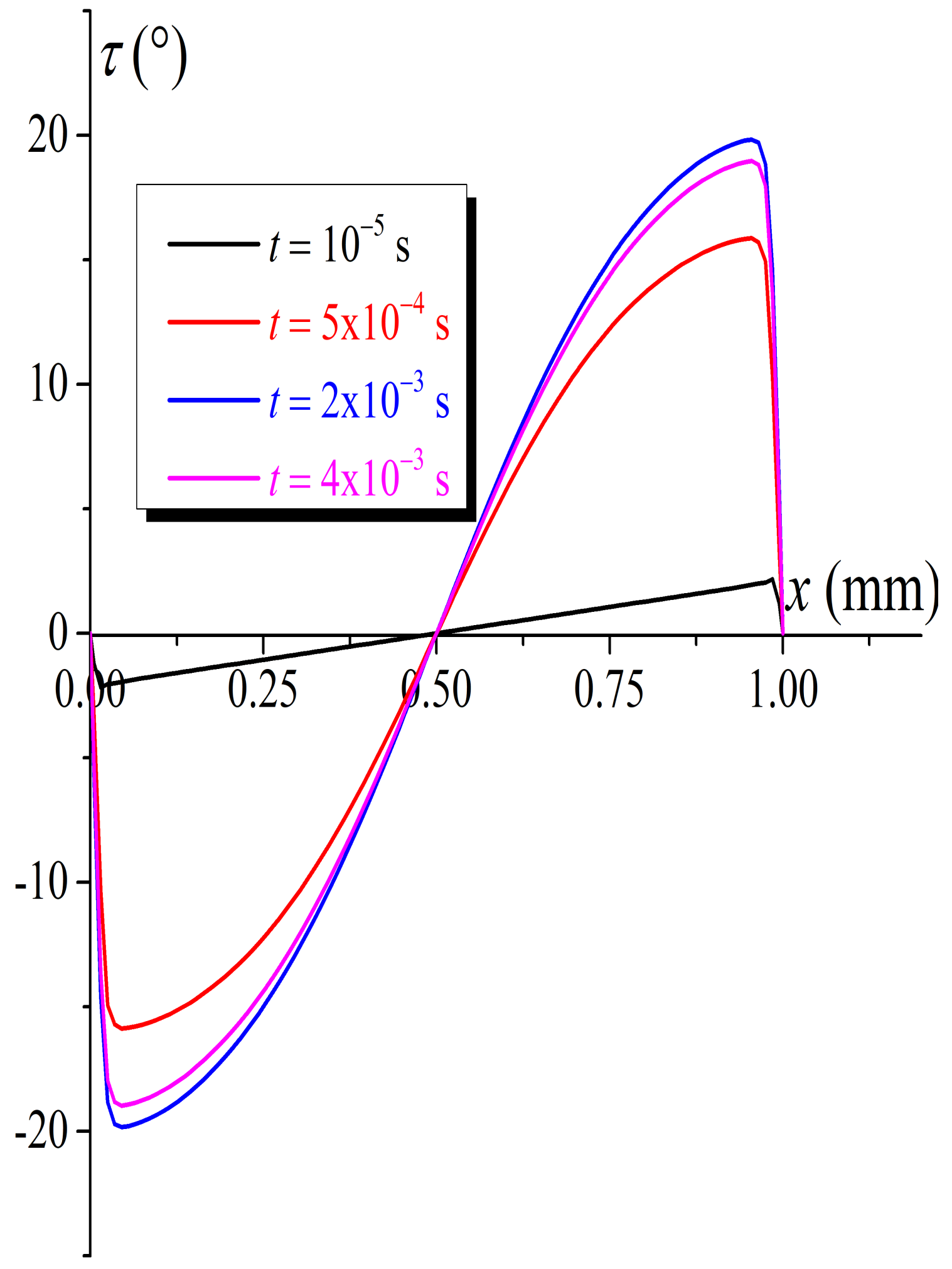

Figure 6: Distribution of the microtemperatures $\tau$ for different instances. 


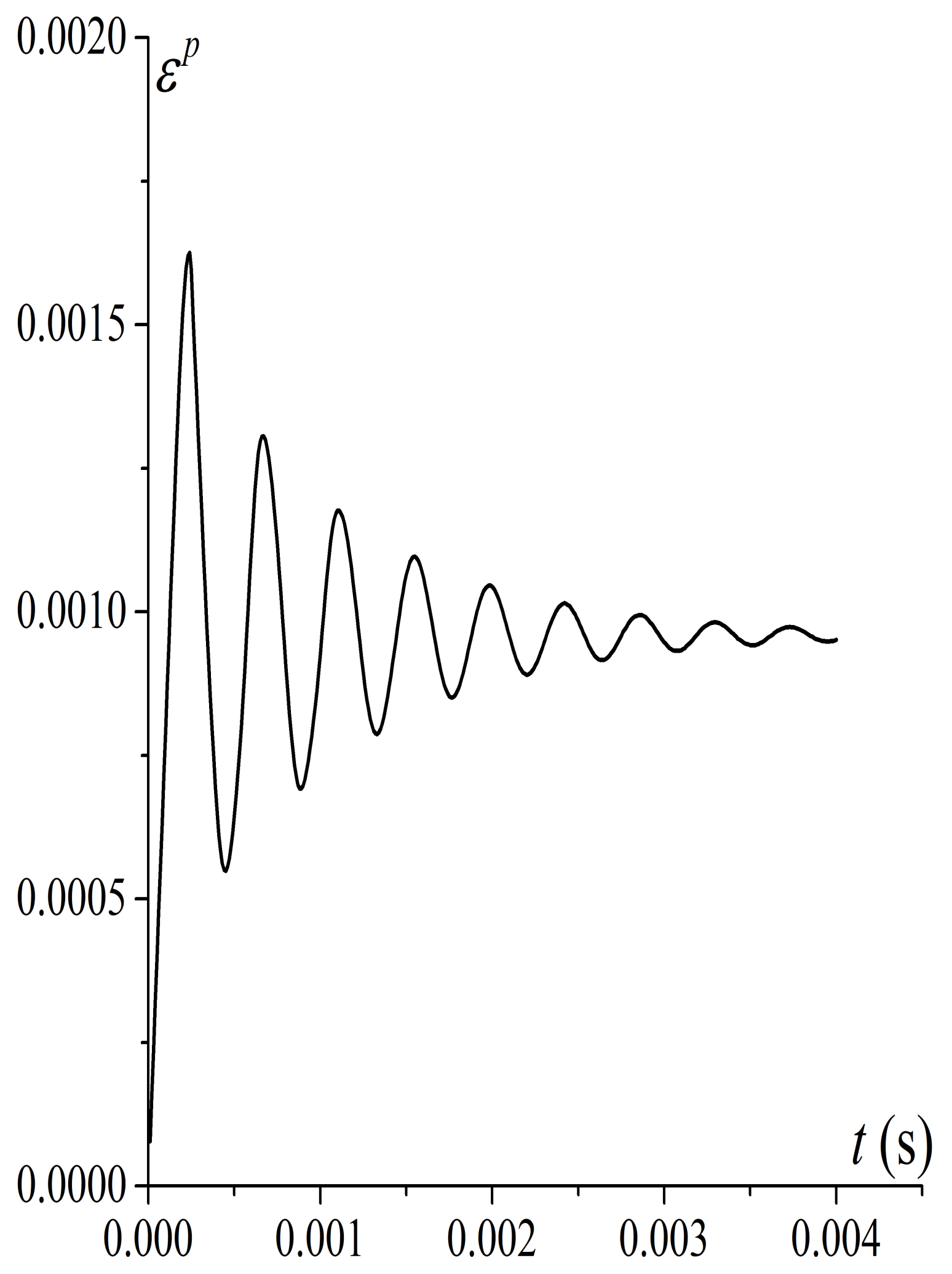

Figure 7: The time evolution of the plastic strain $\varepsilon^{p}$ at the middle of the bar. 


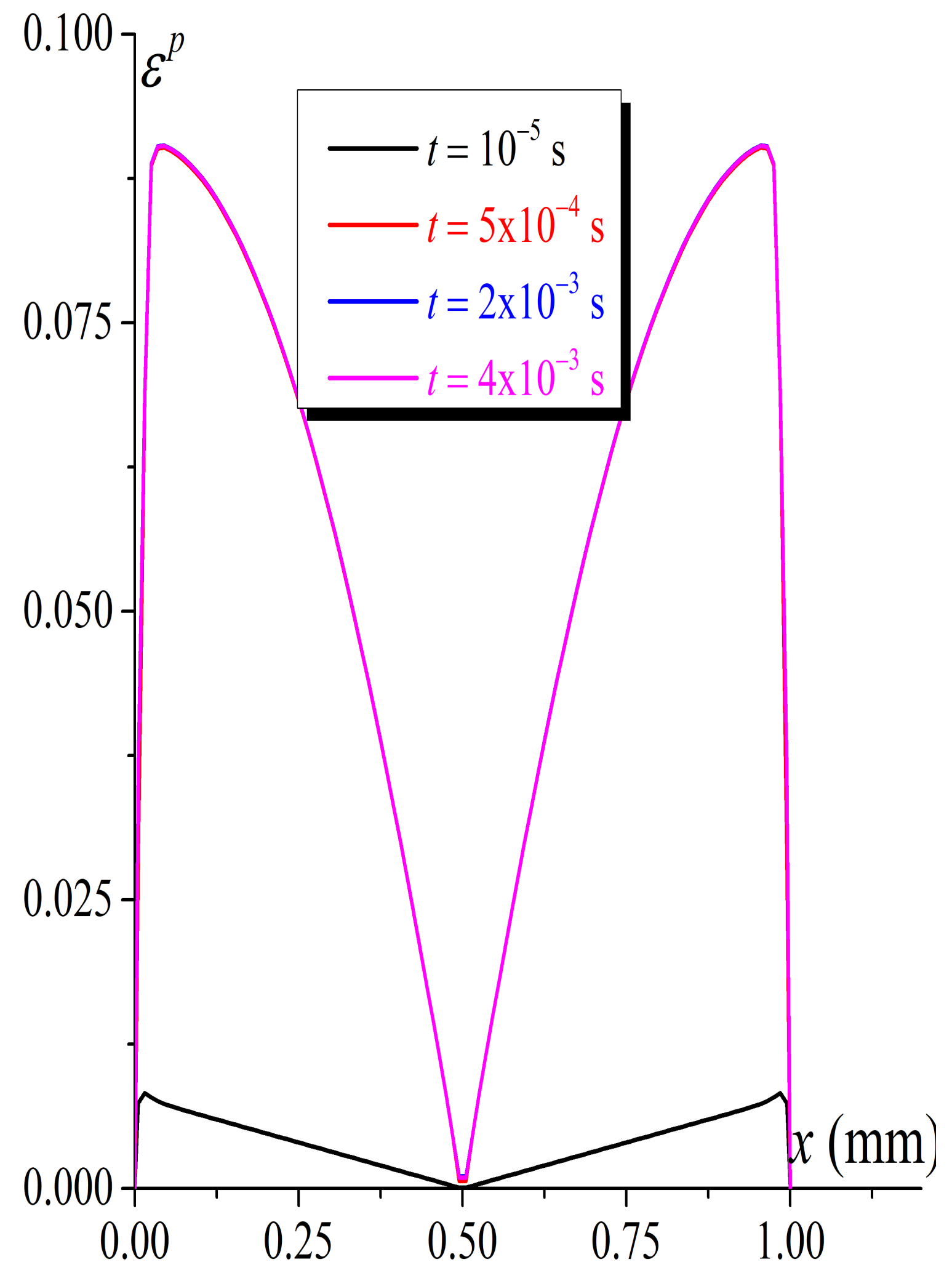

Figure 8: Distribution of the plastic strain $\varepsilon^{p}$ for different instances. 


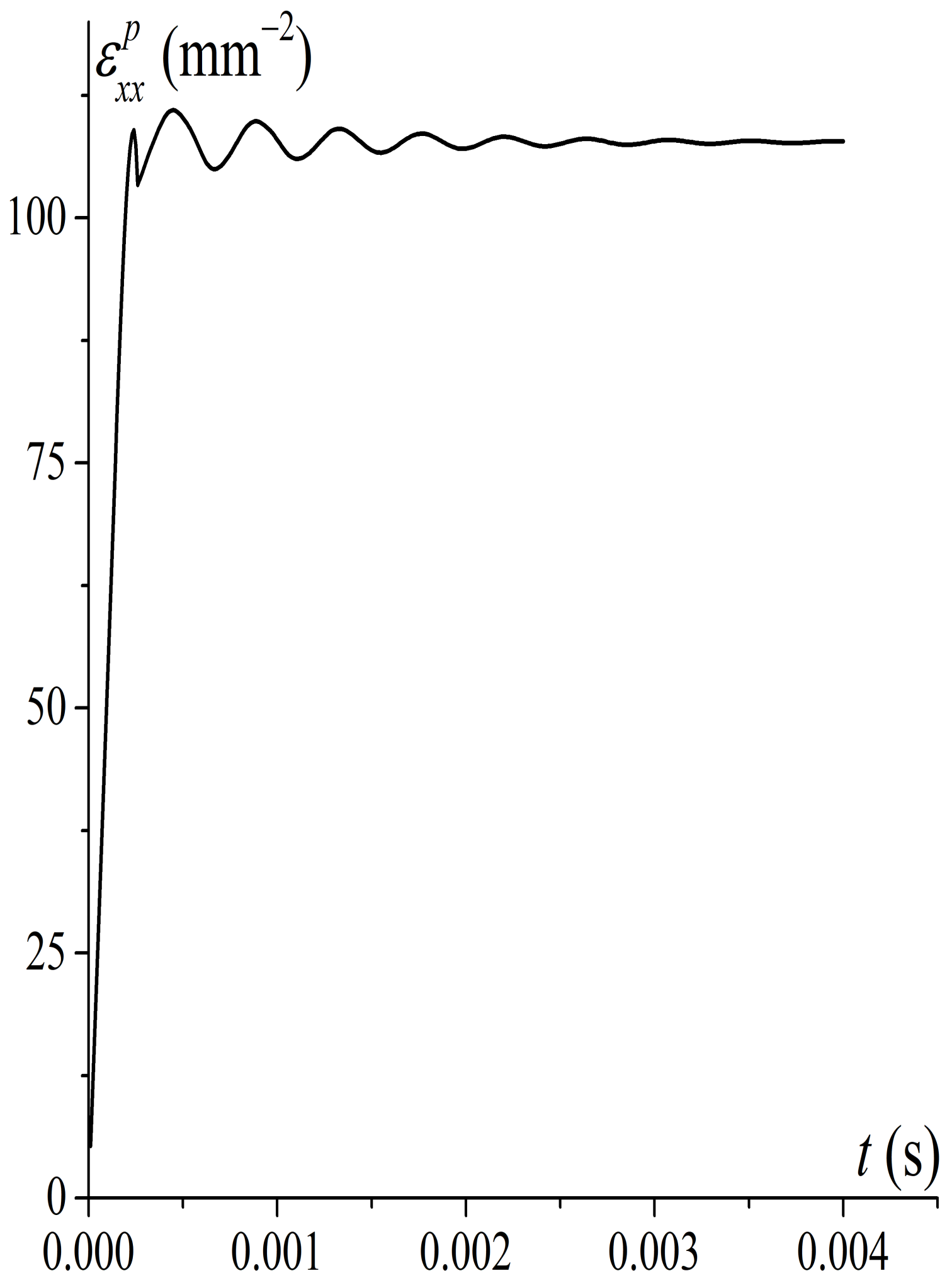

Figure 9: The time evolution of the Laplacian of the plastic strain $\varepsilon_{x x}^{p}$ at the middle of the bar. 


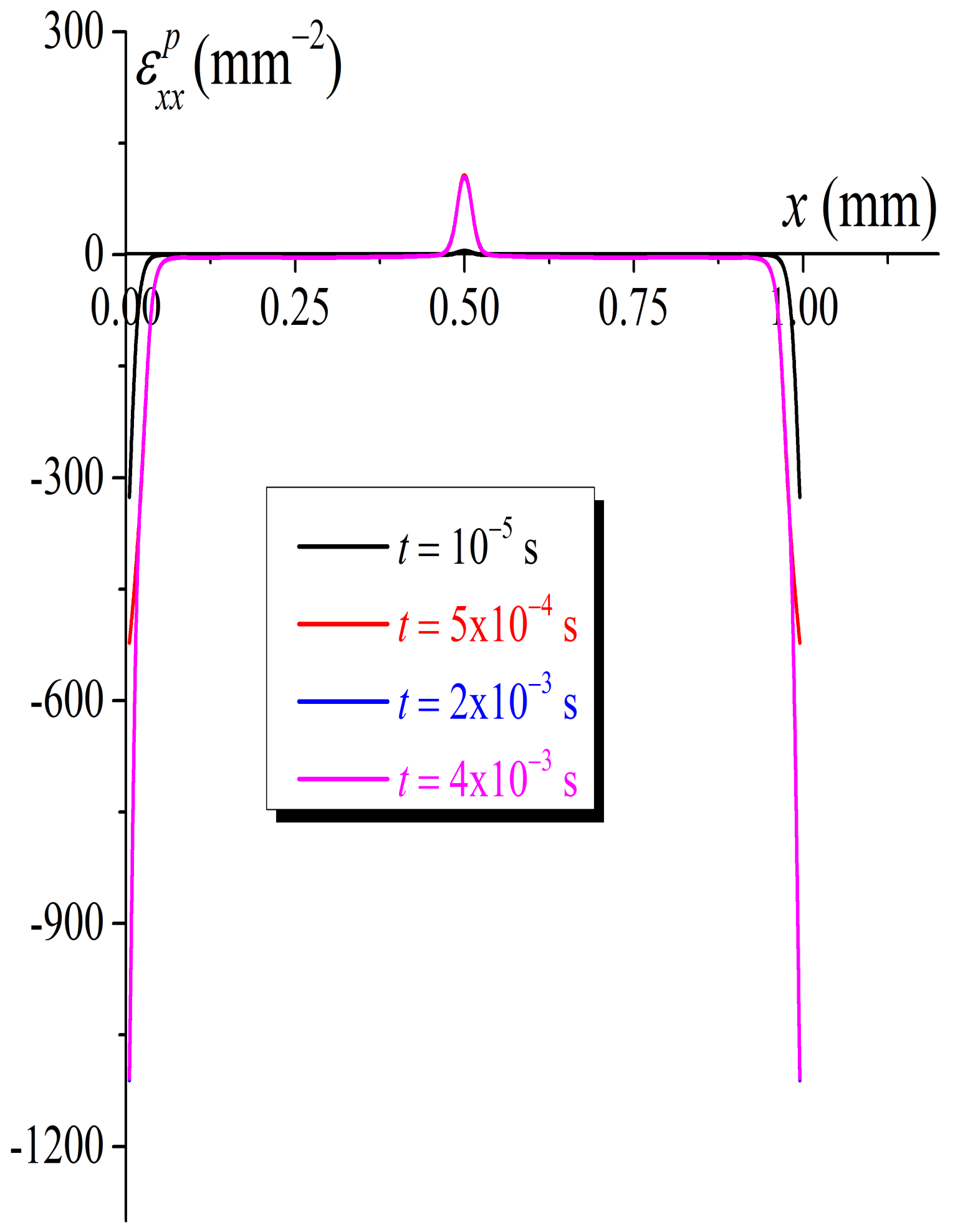

Figure 10: Distribution of Laplacian of the plastic strain $\varepsilon_{x x}^{p}$ for different instances. 


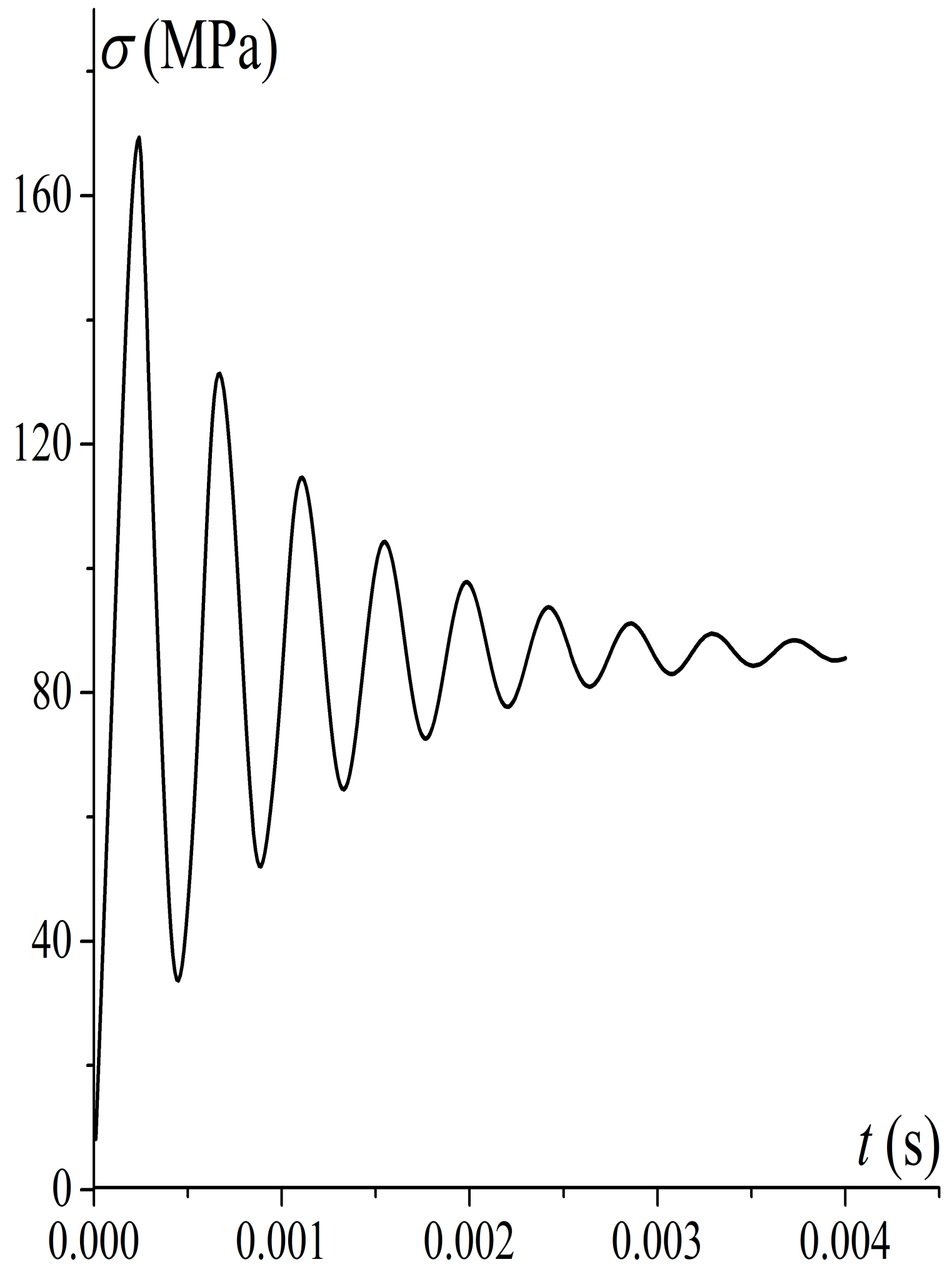

Figure 11: The time evolution of the stress $\sigma$ at the middle of the bar. 


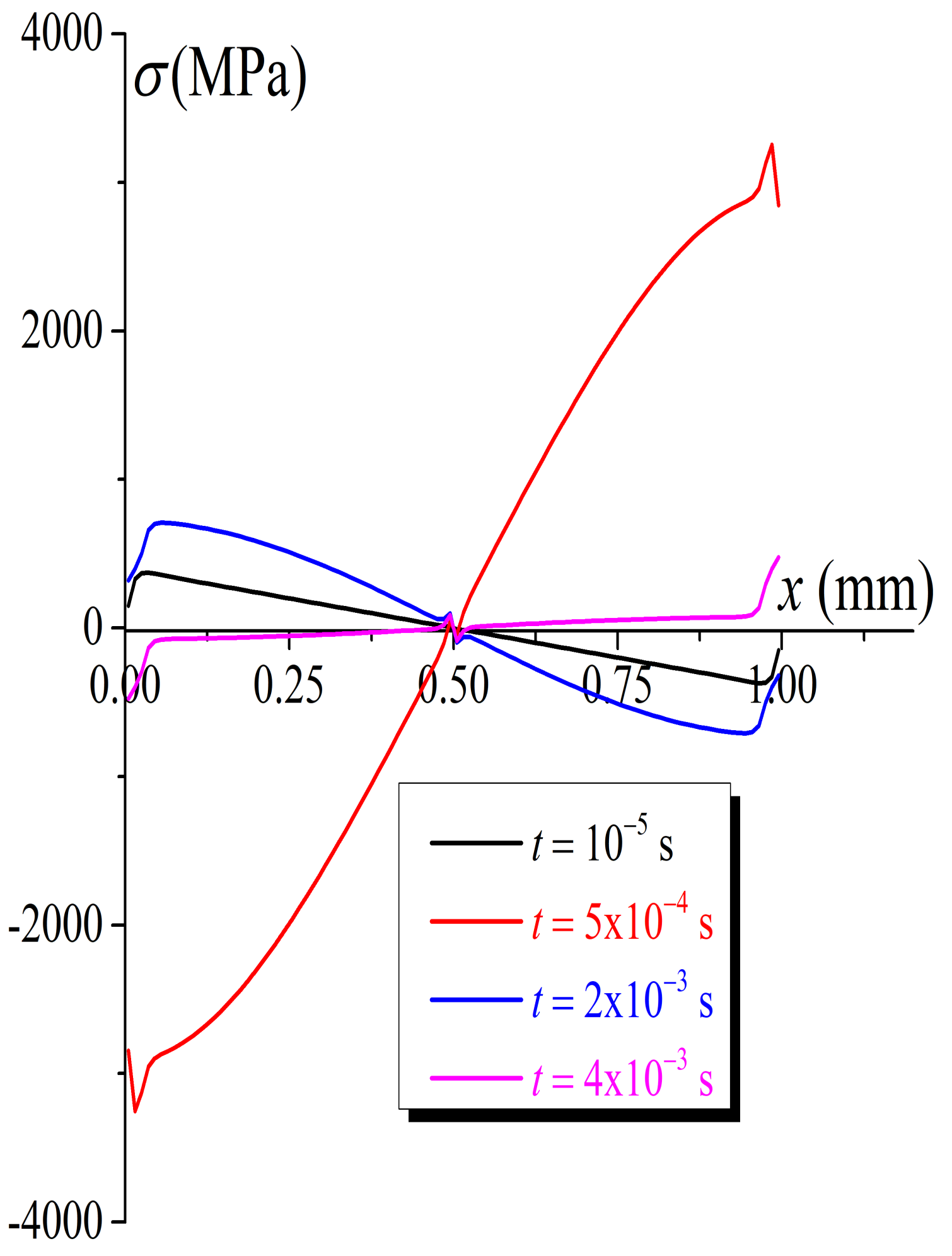

Figure 12: Distribution of the stress $\sigma$ for different instances. 


\section{Conclusion}

We summarize the obtained results as follows:

(i) In this paper we have derived a thermo-gradient-dependent theory of plasticity with microtempartures by including both the second-order strain gradient terms in the stress-strain law and the microtemperatures concept into the second law of thermodynamics. This work can be considered as a continuation or an extension of a previous work [6] where the microtemperatures were not taken into account. By comparison with other gradient-dependent plasticity models [10, 25, 32, 40, the model proposed in this paper is more reasonable in predicting the propagation of thermal, microtemperatures and elastoplastic waves. This work, which has not been obtained in any reference yet, represents a first step towards understanding the fundamental limits of intrinsic thermal and microtemperatures dissipations in gradient-dependent plastic materials.

(ii) By means of semigroup theory, the well-posedness of the thermo-gradient-dependent plasticity for a one-dimensional problem was proved. The analyticity and exponential stability issues were discussed as well. We have proved that, in the one-dimensional case, the corresponding semigroup $S(t)$ is not analytic, but the exponential stability of solutions is kept. For the special case where $h^{g}=0$ and the other coefficients of the system are kept non-zero, the analyticity of solutions holds. The importance of the multi-dimensional counterpart of these results is clear, but this question is difficult from a mathematical point of view and remains open. The important question is whether or not this exponential stability will be preserved in multi-dimensional problems. We have the coupling between a strong conservative equation due to the presence of higher gradients with two dissipative equations. It is interesting to know if the raised coupling is so strong that the dissipation terms bring the system to the exponential decay in multi-dimensional problem, even if they are global and not local or at the boundary. Furthermore, in the multi-dimensional case the situation is different from the one-dimensional setting. In that case, the number of degrees of freedom of the displacement field vector is greater than the one of the temperature. This means that without additional damping mechanisms, the given dissipations are not strong enough to ensure analyticity of solutions to higher-dimensional problems. Moreover, as the semigroup is not analytical in one-dimensional setting, one would expect it not to be in a multi-dimensional setting.

Furthermore, the extension of the one-dimensional finite element approach to a multi-dimensional case (two-dimensional or three-dimensional) seems to be technically complicated. In fact, the Matlab code that we have developed for the one-dimensional case requires to be significantly modified to solve multi-dimensional problems. In the latter case, new types of finite elements, which account for strain gradient aspects, need to be formulated and developed within this code (shell or solid elements). Also, more elaborate numerical techniques must be implemented to efficiently manage some numerical issues (e.g., how to integrate the coupled constitutive equations, how to assemble the elementary stiffness and mass matrices, and how to solve the global matrix system). Hence, the extension of the current contribution (mathematical and numerical developments) to multi-dimensional case will the matter of future contributions.

The results presented in this paper should prove useful for researchers in materials science, designers of new 
materials, low-temperature physicists, as well as for those working on the development of higher-order gradient theories. In particular, the numerical schemes will be useful in simulation and identification studies to predict and better understand the structural and thermal responses of such systems.

\section{Acknowledgements.}

Part of this work was done when the first author visited the laboratory LEM3 of ENSAM of Metz as invited Professor, 01-30 April 2019. He thanks them for their hospitality.

ORCID Moncef Aouadi http://orcid.org/0000-0003-3400-5670

\section{References}

[1] R. K. Abu Al-Rub, G. Z. Voyiadjis, D. J. Bammann, A thermodynamic based higher-order gradient theory for size dependent plasticity, Int. J. Solids Struct. 2007, 44, 2888.

[2] J. D. Achenbach, Wave Propagation in Elastic Solids, North-Holland, Amsterdam 1973.

[3] E. C. Aifantis, On the microstructural origin of certain inelastic models, Trans. ASME. J. Eng. Mater. Technol. 1984, 106, 326.

[4] M. S. Alves, J. E. Muñoz Rivera, M. Sepúlveda, O.P. Vera Villagrán, Analyticity of semigroups associated with thermoviscoelastic mixtures of solids. J. Therm. Stress. 2009, 32, 986.

[5] M. Aouadi, F. Passarella, V. Tibullo, Analyticity of solutions to thermoviscoelastic diffusion mixtures problem in higher dimension, Acta Mech. 2020, 231, 1125.

[6] M. Aouadi, M. Ben Bettaieb, F. Abed-Meraim, Mathematical and numerical analysis in thermo-gradientdependent theory of plasticity, Z. Angew. Math. Mech. 2018, 98, 1603.

[7] M. Aouadi, M. Ciarletta, F. Passarella, Thermoelastic theory with microtemperatures and dissipative thermodynamics, J. Thermal Stresses, 2018, 41, 522.

[8] M. Aouadi, Some theorems in the isotropic theory of microstretch thermoelasticity with microtemperatures, J. Thermal Stresses, 2008, 31, 649 .

[9] R. De Borst, H. Mühlhaus, Gradient-dependent plasticity: formulation and algorithmic aspects, Int. J. Num. Meth. Engng. 1992, 35, 521.

[10] R. de Borst, J. Pamin, M.G.D. Geers, On coupled gradient-dependent plasticity and damage theories with a view to localization analysis, Eur. J. Mech. A/Solids, 1999, 18, 939.

[11] P. Casas, R. Quintanilla, Exponential stability in thermoelasticity with microtemperatures. Int. J. Eng. Sci. 2005, 43, 33 . 
[12] A. C. Eringen, Microcontinuum Field Theories. I. Foundations and Solids. Springer, New York, 1999.

[13] A. Fischer, A special Newton-type optimization method. Optimization 1992, 24, 269.

[14] A. Fischer, Solution of monotone complementarity problems with locally Lipschitzian functions. Mathematical Programming, 1997, 76, 513.

[15] J.A. Goldstein, Semigroups of Linear Operators and Applications, Oxford University Press, New York 1985.

[16] R. Grot, Thermodynamics of a Continuum with Microstructure, Int. J. Eng. Sci. 1969, 7, 801.

[17] F.L. Huang, Characteristic condition for exponential stability of linear dynamical systems in hilbert spaces, Ann. Diff. Eqs. 1985, 1, 43.

[18] D. Ieşan, R. Quintanilla, On a theory of thermoelasticity with microtemperatures, J. Thermal Stresses, 2000, 23, 199.

[19] D. Ieşan, R. Quintanilla, On thermoelastic bodies with inner structure and microtemperatures, J. Math. Anal. Appl. 2009, 354, 12.

[20] D. Ieşan, R. Quintanilla, Qualitative properties in strain gradient thermoelasticity with microtemperatures, Math. Mech. Solids, 2017, 5, 240.

[21] D. Ieşan, On a theory of thermoelasticity without energy dissipation for solids with microtemperatures, Z. Angew. Math. Mech. 2018, 98, 870.

[22] G. Jaiani, L. Bitsadze, On basic problems for elastic prismatic shells with microtemperatures Z. Angew. Math. Mech. 2016, 96, 1082.

[23] P. M. Jordan, P. Puri, Thermal stresses in a spherical shell under three thermoelastic models, J. Thermal Stresses, 2001, 24, 47.

[24] Z. Liu, S. Zheng, Semigroups Associated with Dissipative Systems, Vol. 398, Res. Notes Math., Chapman \& Hall/CRC, Bocca Raton, FL 1999.

[25] H. Lyu, N. Taheri-Nassaj, H. M. Zbib, A multiscale gradient-dependent plasticity model for size effects, Philos. Mag. 2016, 96, 1883.

[26] A. Magaña, R. Quintanilla, Exponential stability in type III thermoelasticity with microtemperatures, Z. Angew. Math. Phy. 2018, 69, 129.

[27] J. E. Muñoz Rivera, M. Sepúlveda, O. Vera, About analyticity for the coupled system of linear thermoviscoelastic equations, Appl. Math. Comput. 2015, 270, 943. 
[28] P. M. Naghdi, J. A. Trapp, The significance of formulating plasticity theory with reference to loading surfaces in strain space, Int. J. Engng. Sci. 1975, 13, 785.

[29] W. Nowacki, Dynamical Problems of Thermodiffusion in Elastic Solids, Proc. Vib. Prob. 1974, 15, 105.

[30] A. Pazy, Semigroups of Linear Operators and Applications to Partial Differential Equations. Vol. 44 of Applied Mathematical Sciences, Springer-Verlag, New York 1983.

[31] P. Riha, On the theory of heat-conducting micropolar fluids with microtemperatures, Acta Mech. 1975, 23, 1 .

[32] L. J. Sluys, R. De Borst and H. B. Mühlhaus, Wave propagation, localization and dispersion in a gradientdependent medium, Int. J. Solids Struct. 1993, 30, 1153.

[33] Y. Song, G. Z. Voyiadjis, Small scale volume formulation based on coupled thermo-mechanical gradient enhanced plasticity theory, Int. J. Solids Struct. 2018, 134, 195.

[34] G. Z. Voyiadjis, D. Faghihi, Thermo-mechanical strain gradient plasticity with energetic and dissipative length scales, Int. J. Plasticity, 2012, 30-31, 218.

[35] G. Z. Voyiadjis, Y. Song, Effect of passivation on higher order gradient plasticity models for nonproportional loading: energetic and dissipative gradient components, Philo. Magazine, 2017, 97, 318.

[36] G. Z. Voyiadjis, Y. Song, T. Park, Higher order thermo-mechanical gradient plasticity model with energetic and dissipative components, J. Engng. Mat. Techn. 2017, 139, 021006-1.

[37] Cz. Wozniak, Thermoelasticity of the bodies with microstructure, Arch. Mech. Stos. 1967, 19, 335.

[38] Cz. Wozniak, Thermoelasticity of non-simple oriented materials, Int. J. Eng. Sci. 1967, 5, 605.

[39] H. Zbib, E.C. Aifantis, On the localization and post-localization behavior of plastic deformation. Part I. On the initiation of shear bands; Part II. On the evolution and thickness of shear bands; Part III. On the structure and velocity of Portevin-Le Chatelier bands, Res Mech. 1989, 261, 279 and 293.

[40] H. W. Zhang, B. A. Schrefler, Gradient-dependent plasticity model and dynamic strain localisation analysis of saturated and partially saturated porous media: one dimensional model, Eur. J. Mech. A/Solids, 2000, $19,503$. 